\title{
Integrated Pan-Cancer Analysis Reveals Distinct Clinical, Genomic and Immunological Features of the LILRB Immune Checkpoint Family in Acute Myeloid Leukemia
}

\section{Zi-jun Xu}

Affiliated People's Hospital of Jiangsu University

Xin-long Zhang

Affiliated Danyang Hospital of Nantong

Ye Jin

Affiliated People's Hospital of Jiangsu University

Shi-sen Wang

Affiliated People's Hospital of Jiangsu University

Yu Gu

Affiliated People's Hospital of Jiangsu University

Ji-chun Ma

Affiliated People's Hospital of Jiangsu University

Xiang-mei Wen

Affiliated People's Hospital of Jiangsu University

Jia-yan Leng

Affiliated People's Hospital of Jiangsu University

Zhen-wei Mao

Affiliated People's Hospital of Jiangsu University

Jiang Lin ( $\square$ linjiangmail@sina.com )

Affiliated People's Hospital of Jiangsu University https://orcid.org/0000-0002-4704-9157

Jun Qian

Affiliated People's Hospital of Jiangsu University

\section{Research Article}

Keywords: LILRBs, immune checkpoint genes, immune evasion, pan-cancer, leukemia

Posted Date: September 28th, 2021

DOI: https://doi.org/10.21203/rs.3.rs-810313/v1 
License: (c) (i) This work is licensed under a Creative Commons Attribution 4.0 International License. Read Full License 


\section{Abstract \\ Background}

Leukocyte immunoglobulin (Ig)-like receptor Bs (LILRBS), a family of type I transmembrane glycoproteins, are known to inhibit immune activation.

\section{Methods}

We comprehensively evaluated the transcriptional levels and prognostic significances of LILRB members in a broad spectrum of cancer types, focusing on its role in AML. In addition, we systematically characterized the genomic and immune landscape in AML patients with altered LILRBs expression.

\section{Results}

Here, we show that $L I L R B s$ were significantly dysregulated in a number of cancers, especially in acute myeloid leukemia (AML). Clinically, high expression of LILRB1-LILRB4 predicted poor survival in six independent AML cohorts. Genetically, LILRB1 was associated with more mutational events than other $\angle I L R B$ members, and multiple genes involving in immune activation were deleted in $L I L R B 1$-high patients. Epigenetically, LILRB4 was significantly hypomethylated and marked by MLL-associated histone modifications in AML. Immunologically, LILRBs were positively associated with monocytic cells including M2 macrophages, but were negatively associated with tumor-suppressive CD8 T cells.

\section{Conclusions}

Our findings reveal critical immunological and clinical implications of LILRBs in AML, and indicate that LILRBs may represent promising targets for immunotherapy of AML.

\section{Background}

Acute myeloid leukemia (AML) is a highly fatal hematopoietic malignancy marked by various cytogenetic and molecular abnormalities and variable responses to treatment [1-3]. Currently, the mainstay of treatment for AML is cytotoxic chemotherapy [4], yet chemoresistance and relapse are commonly seen in in clinical practice. Some regimens under study, such as the combination of hypomethylating agents (HMAs) and Venetoclax, have shown promising results in certain subsets of AML patients $[5,6]$. However, there remains an urgent need to develop novel effective therapies for various subsets of AML.

Noteworthy, immune checkpoint inhibitors (e.g., anti-PD-1 and anti-PD-L1 antibodies) have revolutionized cancer treatment during the past decade in treating cancers such as non-small-cell lung carcinoma and melanoma [7, 8]; however, the transfer of immunotherapy to AML has been less 
successful than to other cancers [9]. Indeed, the AML microenvironment is predominantly immunosuppressive. For example, we have previously demonstrated that M2 macrophages, a classical immunosuppressive component, was preferentially enriched in AML than other hematological malignancies and normal controls [10]. Also, a recent single-cell RNA-seq study has reported proportionally fewer T cells and CTLs in AML than normal controls, and the function of these $\mathrm{T}$ cells are profoundly impaired, probably mediated by CD14 + monocyte-like cells [11, 12]. Moreover, Noviello et al. have reported that bone marrow $\mathrm{T}$ cells at AML relapse showed an exhausted phenotype, which was absent in patients maintaining long-term complete response [13]. These findings suggest encouraging therapeutic opportunities by modulating the immune environment in AML.

The leukocyte immunoglobulin (Ig)-like receptor subfamily B (LILRB) proteins are a group of type I transmembrane glycoproteins with extracellular Ig-like domains that bind ligands and intracellular immunoreceptor tyrosine-based inhibitory motifs (ITIMs) [14]. This group of receptors contains 5 members (LILRBT-LILRB5) that are mainly expressed in hematopoietic lineage cells and also various types of tumors [14]. As these proteins negatively regulate immune activation [15-17], they are often considered as immunosuppressive component in the tumor microenvironment (TME). In AML, the TMEmodulating role of $L I L R B s$ have recently came into focus, especially for LILRB4. As demonstrated by the Gui group that $L I L R B 4$ facilitates tissue infiltration of AML cells by substantially suppressing $T$ cell activities, and blocking LILRB4 activity efficiently inhibited AML development in vitro and in vivo $[18,19]$. In addition, $L I L R B 1$, which showed predominant expression in monocytic AML cells as LILRB4 did [20], was found to be up-regulated in dysfunctional CD8 + T cells from AML than T cells from healthy controls [21]. Interestingly, a non-immunological AML-promoting role was reported for $L I L R B 2$, which binds Angpt/2 to maintain stemness of normal stem cells and support leukemia development by inhibiting differentiation of AML cells [22]. Despite the functional importance of LILRBs in cancers, there currently lacked a systematic study to explore the expression patterns and clinical implications of all LILRB members in pan-cancers, especially in AML. Therefore, in this study, drawing on rich multi-omics data in the public domain, we comprehensively evaluated the transcriptional levels and prognostic significances of $L I L R B$ members in a broad spectrum of cancer types, focusing on its role in AML. In addition, we systematically characterized the genomic and immune landscape in AML patients with altered LILRBS expression.

\section{Materials And Methods}

\section{Analysis of gene expression data}

Briefly, the mRNA expression data of LILRB family in normal tissues were obtained from the GenotypeTissue Expression (GTEx) project (www.gtexportal.org/) [23]. To confirm the expression patterns of LILRBs in normal tissues, we then explored the HPA (Human protein atlas) and FANTOM5 dataset from the human protein atlas database (http://www.proteinatlas.org/) [24]. Expression data of LILRBs for over 1000 cancer cell lines from various organ sites were accessed through Cancer Cell Line Encyclopedia (CCLE) (https://www.broadinstitute.org/ccle) [25]. Furthermore, RNA-seq data of 64 cell lines from The 
Human Protein Atlas (HPA) ((https://www.proteinatlas.org/) [24] were used to validate expression patterns of LILRBs in cancer cell lines.

To determine the expression patterns of LILRBs between tumor and adjacent normal tissues across a broad range of cancer types, we systematically analyzed the gene expression data of 9465 tumor and 7831 normal samples based on RNA sequencing data from the TCGA and the GTEx projects. All these datasets were downloaded from the UCSC Xena project and were normalized between arrays using the limma package [26]. To validate the differential expression of $L I L R B s$ between AML and normal controls, we further retrieved two datasets containing both healthy and $A M L$ samples from Gene Expression Omnibus (GEO) (https://www.ncbi.nlm.nih.gov/geo/) (accession number GSE63270 and GSE30029). We also used a gene-expression dataset of normal hematopoietic cells (GSE42519) to assess the expression patterns of $L I L R B$ family genes at various stages of normal hematopoiesis.

We used the Hemap dataset curated by Dufva et al. [27], which includes datasets of AML, pre-B-ALL, DLBCL, and MM, to analyze the association between LILRBs expression and common molecular subtypes. The molecular subtypes were defined as previously described [27]. Three datasets-BeatAML, TCGA, and GSE13159-which have detailed cytogenetic information, were used to determine whether LILRB4 expression was associated with MLL-rearranged AML. The counts per million (CPM) table of BeatAML were downloaded from the Supplementary Materials from Tyner et al. [28]. LILRB4 expression level were log2 $(1+$ CPM) transformed before visualization. We computed single-sample GSEA (ssGSEA) enrichment scores for four MLL-r-related gene signatures from MSigDB (http://www.gseamsigdb.org/gsea/msigdb/) using the R 'GSVA' package.

\section{Analysis of AML single-cell RNA-sequencing (scRNA-seq) data}

For single cell RNA-seq (scRNA) data analysis, previously published scRNA-seq data from $16 \mathrm{AML}$ samples at diagnosis consisting of 30,712 bone marrow (BM) cells (Van Galen AML scRNA) were downloaded from GEO (GSE116256) [11], another scRNA-seq data for 8 patients consisting of 30,579 AML BM cells (FIMM AML scRNA) were retrieved via the Synapse Web Portal (https://www.synapse.org and doi: $10.7303 /$ syn21991014). Data was processed and visualized using custom scripts provided by Dufva et al. [27].

\section{Analysis of genetic alteration data}

The genetic alterations of LILRBs from TCGA PanCancer Atlas studies (10967 patients), including somatic mutations, amplification, and deep deletion were assessed through the cbioportal for Cancer Genomics (http://www.cbioportal.org). To determine the association between LILRBs expression and common gene mutations, patients from the TCGA LAML cohort were first stratified into two groups by the median expression value of respective member genes, then mutation status for 24 most frequently mutated genes were identified using TCGA mutational data. The relationships between mutation status and $L I L R B s$ expression were analyzed by two-sided Fisher exact tests. The mutational profiles of patients 
with high or low $L I L R B 1 / L I L R B 5$ expression were displayed as co-bar plots using the "Maftools" package [29]. To detect copy number alterations (deletions and amplications) in high- and low-LILRB1 expressers, we analyzed filtered segmented copy number data in each group (Affymetrix SNP 6.0 platform) using the GISTIC 2.0 algorithm [30].

\section{Analysis of gene methylation data}

For comparison of methylation status of LILRBs between tumor and normal samples, beta values of Illumina $450 \mathrm{k}$ probes at the promoter region of five genes were retrieved by DiseaseMeth version 2.0 web portal (http://bio-bigdata.hrbmu.edu.cn/diseasemeth/analyze.html). Correlation between LILRBS methylation with expression and survival data across cancers were analyzed through the GSCALite platform [31]. For AML, the DiseaseMeth dataset contains methylation data of 271 AML (from TCGA, GSE62303, and GSE64934) and 10 normal samples (from GSE58477). Another methylation datasetGSE63409-contains DNA methylation profiles of 20 leukemia stem cells, 24 blast cells and 30 normal hematopoietic stem and progenitor cells. Beta values of Illumina $450 \mathrm{k}$ probes nearest the transcription start site of the genes were selected to represent methylation level of the gene promoter area. Heatmaps displaying methylation levels of LILRBS across samples were generated using 'pheatmap' package.

\section{Analysis of Chromatin immunoprecipitation-sequencing (ChIP-seq) data}

ChIP-seq data for MLL-fusion proteins and three histone marks (H3K79me2, H3K27ac, and H3K4me3) from MV4-11 and THP-1 cells were obtained from GSE79899 [32]. For validation purpose, the three epigenetic marks from five other ChIP-seq datasets (H3K79me2 from GSE82116 and GSE71779; H3K27ac from GSE89336 and GSE71776; H3K4me3 from GSE61785 and GSE82116) were also included in our analyses. The gene tracks were generated by uploading the wiggle files as custom tracks onto the UCSC Genome Browser, assembly hg19. Wiggle files that have failed to be loaded as custom tracks were first converted to bigwig using the UCSC wigToBigWig tool.

\section{Survival Analysis}

We used the "Gene Outcome" module of TIMER2.0 (http://timer.cistrome.org/) [33] to investigate the association between the expression of $L I L R B$ members and clinical outcomes across 33 cancer types. The association between transcript levels of $L I L R B$ members and overall survival (OS) across cancers were assessed by univariate Cox regression. To confirm the prognostic value of $L I L R B s$ in AML, we further obtained five independent GEO datasets (GSE10358, $n=304$; GSE37642 [U133A], $n=422$; GSE37642 [U133plus2], $n=140$; GSE106291, $n=250 ;$ GSE71014, $n=104$ ) with available survival information. AML patients from these datasets and the TCGA dataset were divided into those with high and low gene expression, according to the optimal cut-off determined by the X-tile method [34]. We then performed Kaplan-Meier analysis (log-rank test) to compare the survival differences of two groups regarding overall survival (six datasets) and event-free survival (EFS) (only in TCGA dataset).

\section{Immune response analysis}


The relative abundances of 22 immune cell populations in AML patients were estimated using the CIBERSORT algorithm as previously described [10]. As CIBERSORT may not be suitable for the use of the RNA-seq data [35], this algorithm was exclusively applied to the TCGA LAML microarray dataset. For validation purpose, the relative fractions of immune cells were also estimated in two relatively large GEO datasets: GSE10358 and GSE6891. In addition, we used other deconvolution methods to quantify the proportions of monocytes (quanTIseq, MCP-counter, CIBERSORT abs, and xCell) and CD8 T cells (EPIC, TIMER, quanTIseq, MCP-counter, CIBERSORT abs, and $x$ Cell). These methods have been integrated as a unified interface by Sturm et al. [36] and are freely available through the TIMER 2.0 web portal (http://timer.comp-genomics.org/).

We evaluated the relationship between $L I L R B s$ and several notable immune checkpoint genes describe by De Simone et al. [37]. Spearman correlation analysis was used to test the association between LILRBS expression and these parameter estimates.

\section{Differential gene expression analysis and functional enrichment analysis}

Differential gene expression analysis for RNA sequencing data was performed using the raw read counts with the R/Bioconductor package "DESeq2", controlled for the false discovery rate (FDR) by the Benjamini-Hochberg procedure. Gene Ontology (GO) analysis and Kyoto Encyclopedia of Genes and Genomes (KEGG) pathway analysis of LILRB1-coexpressed genes were performed using the STRING database (http://www.string-db.org/). GO and KEGG terms with false discovery rate (FDR)-corrected $p$ values less than 0.05 were considered as significantly enriched. For displaying purposes, the top $10 \mathrm{GO}$ terms of each three GO categories-biological process (BP), cellular component (CC), and molecular function (MF), and the top 10 KEGG pathway terms were visualized as bar plots.

\section{Protein-protein interaction (PPI) network analysis}

We applied STRING (http://string.embl.de/) to construct a protein-protein interaction (PPI) network of the differentially expressed genes (DEGs). We chose a confidence score $>0.9$ as the judgment criterion. Cytoscape visualization software (version 3.6.1) was used to present the LILRB1-related sub-network.

\section{Gene Set Enrichment Analysis (GSEA)}

Gene set enrichment analysis (GSEA) was performed on the TCGA dataset using GSEA v4.1.0 software (http://www.broad.mit.edu/gsea). Statistical significance of GSEA results was determined by 1,000 gene set permutations, with signal-to-noise gene ranking. All the gene sets used in this study were obtained from GSEA MSigDB website (http://software.broadinstitute.org/gsea/msigdb/index.jsp), and the gene sets were considered to be significantly enriched at a false discovery rate $<0.25$ and normalized $P$-value $<$ 0.05 . Three categories of gene sets were used in this study: $\mathrm{C} 2$, curated gene sets containing genes coregulated in response to specific perturbations; $\mathrm{C7}$, immunologic signature gene sets that represent cell states and perturbations within the immune system; and $\mathrm{H}$, hallmark gene sets which represent welldefined biological states or processes. 


\section{Statistical analysis and visualization}

Wilcoxon rank sum tests were used to compare differences between two groups. Specifically, for Fig. 6a, we determined differential expression of LILRBs between each molecular subtype and the remaining samples for each disease subtype. The average fold changes (FCs) and Bonferroni-adjusted p-values (false discovery rate [FDR]) were computed using Wilcoxon rank sum tests. The FDR values were then combined for each $L I L R B$ members using Stouffer's method and categorized into five groups based on significance cutoffs for visualization $(0.05,0.01,0.001,1 e-5,1 e-16)$. All statistical analyses and visualizations were performed using either indicated web servers or $\mathrm{R}$ version 4.0.4. Specifically, the expression map of LILRBS was generated using the "gganatogram" package [38], the box, bar, scatter and bubble plots were produced with the R package "ggplot2", "ggpubr" and "ggsci", the volcano plots were generated using the "EnhancedVolcano" package, and survival curves were made using the "survival" package. The correlation matrix was calculated and visualized using the "corrplot" or "ggcor" library. Finally, "circlize" was used to create the chord diagrams. All statistical tests were two-sided with p-values less than 0.05 considered significant.

\section{Results}

\section{Expression patterns of LILRBs in normal tissues and cancer cell lines}

In our first attempt, the expression patterns of $L I L R B s$ in different human tissues were determined based on RPKM values using GTEx [39] (http://www.GTExportal.org/home/). We note that the highest expression of LILIBS were observed in spleen, followed by blood and the lung tissue, while in other tissues, they were only weakly expressed (Fig. 1a and Supplementary Figure S1). Importantly, the preferential enrichment of LILRBs in spleen was further validated in the FANTOM5 and HPA (Human protein atlas) dataset (Supplementary Figure S2 and S3). Next, we explored the expression profiles of LILRBs in cancer cell lines from Cancer Cell Line Encyclopedia (CCLE). As shown in Fig. 1b and Supplementary Figure S4, LILRBs showed relatively high expression in cell lines of malignant hematological cell lines, such as acute myeloid leukemia (AML), acute lymphocytic leukemia (ALL), lymphomas, and multiple myeloma (MM). We also analyzed the expression levels of LILRBs based on RNA-seq in 64 different cell lines, as part of the Cell Atlas in the HPA. This analysis confirmed that LILRBS was highly expressed in cell lines of the myeloid and lymphocytic origin (Supplementary Figure S5). Notably, LILRB1 and 2 showed higher expression in monocytes and the THP1 monocyte cell line. Together, these findings indicated a cellular-, tissue-, and disease- specificity of LILRBs expression.

\section{Analysis of LILRB family gene expression levels in tumor and non-tumor tissues}


Previous studies revealed that members of the LILRB family were upregulated in a number of cancers [40, 41]. Here, using pan-cancer datasets from the TCGA project, we found that the expression levels of LILRB1-LILRB5 were positively correlated with each other (Fig. 1c), suggesting they may share some common features or functions; of them LILRB5 showed relatively weak correlation with the other four genes. Combining the normal tissue of the GTEx dataset as controls, we then systematically compared LILRBs expression between tumor and adjacent normal tissue across 28 cancer types ( 9465 tumor and 7831 normal samples). Surprisingly, LILRBs were significantly dysregulated in almost all cancer types (Fig. 1d and Supplementary Figure S6). For $L I L R B 1, L I L R B 2$, and $L I L R B 4$, increased expression in tumors was more commonly seen; whereas $L I L R B 3$ and $L I L R B 5$ were significantly down-regulated in the majority of cancer types (Fig. 1d and e). For LILRB1-LILRB4, the most remarkable difference was observed between AML and normal its normal counterparts (Fig. 1d and Supplementary Figure S6). We also found that LILRB1-LIRB4 were highly expressed in glioblastoma multiforme (GBM), head and neck squamous cell carcinoma (HNSC), kidney renal clear cell carcinoma (KIRC), kidney renal papillary cell carcinoma (KIRP), pancreatic adenocarcinoma (PAAD), and skin cutaneous melanoma (SKCM), whereas they were markedly decreased in adrenocortical carcinoma (ACC), lung adenocarcinoma (LUAD), lung squamous cell carcinoma (LUSC), and thymoma (THYM), as compared with normal controls (Fig. 1d and Supplementary Figure S6).

\section{LILRBs were significantly up-regulated in AML and dynamically expressed during normal hematopoiesis}

Our observations, together with previous findings [18], reflect an AML-specific expression patterns of LILRBs. We thus further explored the differential expression of LILRBs in two independent AML datasets with normal controls available. For these two datasets, only LILRB1 and LILRB3 were consistently upregulated in AML (Fig. 1f and g), whereas LILRB5 was down-regulated (Supplementary Figure S7a and b). Interestingly, LILRB4, which was previous shown to be increased in AML [18], exhibited no difference in expression in the two datasets (Fig. $1 \mathrm{f}$ and g).

We next sought to determine the expression patterns of $L I L R B s$ during normal hematopoiesis using a published data (GSE42519) [42]. We found that the transcripts of LILRBs was relatively high in bone marrow (BM) hematopoietic stem cells (HSCs), with a gradual diminishment when HSC differentiated to committed myeloid progenitors. The expression remained low in megakaryocyte-erythroid progenitor (MEP) and then steadily increased during myeloid maturation, reaching the highest level of expression in the mature polymorphonuclear cell (PMN) (Figure S7c). This dynamic regulation of LILRBs transcripts suggests a potential role of LILRBs in HSC biology.

\section{Landscape of genetic alterations of LILRBs in tumors}

We also investigated genetic alterations (including mutations, amplifications, and deletions) frequencies of $L I L R B s$ across pan-cancers. The average alteration frequencies of five genes were summarized in Fig. 4a and the oncoprint was present in Supplementary Figure S8a. The highest mutation loads of LILRBS was observed in SKCM (31.53\%), followed by uterine corpus endometrial carcinoma (UCEC) 
(14.56\%), LUAD (14.49\%), and LUSC (13.14\%) (Fig. 4a and C). Overall, LILRB1 was the most highly mutated and $L I L R B 3$ the least; the most frequent genomic variants were missense mutations for five genes (Fig. 4b and Supplementary Figure S8b). For diffuse large B-Cell lymphoma (DLBC), mesothelioma (MESO), and THYM, copy number alterations (CNAs) were the only genetic events (Fig. 4a). Amplifications were more commonly seen in cancers such as ACC, uterine carcinosarcoma (UCS), and bladder urothelial carcinoma (BLCA), while deletions were mostly found in cancers like brain lower grade glioma (LGG), ovarian serous cystadenocarcinoma (OV), and testicular germ cell tumor (TGCT) (Fig. 4a and d). In AML where LILRBs expression were markedly changed, however, genetic alteration frequencies of these genes were extremely low, suggesting other mechanisms might contribute to the abnormal LILRBs expression in AML (Fig. 4a, C, and d).

\section{LILRBs were hypomethylated in AML}

We next asked whether DNA methylation regulates the expression of LILRBs in cancers. We first retrieved the methylome data of LILRBS across 30 cancer types with matched controls through the human disease methylation database Diseasemeth version 2.0 (http://bio-bigdata.hrbmu.edu.cn/diseasemeth/).

Surprisingly, we found that $L I L R B$ members were significantly hypomethylated in almost all cancer types analyzed as compared to normal samples (Fig. 3a). We then investigated the correlation between methylation and expression levels of LILRBs using GSCALite [31]. We found that methylation level of $L I L R B 1$ was negatively associated with its mRNA expression in most cancer types (Fig. 3b). For other $L I L R B$ members, methylation and expression levels were also mainly negatively correlated, with only a few positive correlations (Fig. 3b). Importantly, consistent with the increased expression of LILRBs in $\mathrm{AML}$, significantly hypomethylated promoters of $L I L R B s$ were observed in both the Diseasemeth (AML, $\mathrm{n}$ = 271; normal, $n=10$ ) and GSE63409 dataset ( $A M L, n=44$; normal, $n=30)$ (Fig. $3 c$ and $d)$. We then studied the correlation between promoter methylation and expression of LILRBs in TCGA AML dataset. Interestingly, expression of $L I L R B 2, L I L R B 3$, and $L I L R B 4$ correlated negatively with promoter methylation and the most significant correlation was observed for LILRB4 (Fig. $3 \mathrm{~b}$ and e). This observation is consistent with a previous report that decitabine (DAC, a demethylating agent) treatment with AML cells remarkably promoted expression of $L I L R B$ family members, especially LILRB4 [43]. Collectively, these results suggest that DNA hypomethylation might contribute to the activation of LILRB members in AML. Further, analyzing the relation between methylation and survival revealed that hypomethylation of LILRBS predicted worse survival in most cancers (Fig. 3f). In AML, hypomethylation of LILRB4 was associated with adverse outcome and hypomethylation of $L I L R B 5$ with favorable outcome (Fig. 3f).

\section{Adverse prognostic impact of LILRBs in AML}

Next, we used Cox regression analyses to explore the association between LILRBs expression and overall survival (OS) in TCGA pan-cancer datasets. Overall, we found the significance and direction of the prognostic significances varied, depending on the cancer types analyzed. For example, increased expression of $L I L R B$ family members were generally associated with worse OS in KIRC, LAML, LGG, TGCT, THYM, and uveal melanoma (UVM). While in SKCM and metastatic SKCM, the reverse was observed 
(Fig. 4a). Interestingly, a previous study has also performed Cox analyses in TCGA data, showing that LILRB1-LILRB4 negatively impacts the survival of AML patients. It is of particular interest to validate the prognostic value of $L I L R B S$ using Kaplan-Meier methods in larger patient cohorts of AML. To this end, we collected five independent datasets from GEO; X-tile was used to determine the optimal thresholds for each $L I L R B$ members in TCGA and GEO datasets. First, we were able to validate the adverse prognostic impact for LILRBT-LILRB4 in the TCGA cohorts (Fig. 4b), whereas high LILRB5 was associated with favorable outcome (Supplementary Figure S9). Importantly, the prognostic value of LILRB1-LILRB4 also extended to the event-free survival (EFS) endpoint and cytogenetically normal (CN)-AML subsets (Supplementary Figure S10a-c). Furthermore, the adverse prognostic impact of LILRBs was validated in TCGA microarray data $(n=183)$ (Supplementary Figure S10d) and other five independent cohorts of AML patients (GSE10358, $n$ = 304; GSE37642 [U133A], $n=422$; GSE37642 [U133plus2], $n=140 ;$ GSE106291, $n$ = 250; GSE71014, $n=104$ ) (Fig. 5a-e), although in some cases only a trend for shorter OS was observed. For subsequent analyses, we will focus on the role of $L I L R B 1-L I L R B 5$ in $\mathrm{AML}$, due to frequent alterations and high prognostic values of $L I L R B s$ in this malignancy.

\section{LILRB4 is aberrantly overexpressed in MLL-rearranged AML and may be a target of MLL fusion proteins}

We next asked whether LILRBs expression could be associated with specific molecular subtypes in AML. To this end, we examined the expression differences of $L I L R B s$ across published transcriptomic subtypes in the Hemap dataset (including AML, pre-B-ALL, DLBCL, and MM) [27]. As expected, all five LILRB members were more highly expressed in monocyte-like AML, while their expressions were relatively weak in the other three malignancies (Fig. 6a). One unanticipated exception to this overall trend was the strong enrichment of LILRB4 in MLL-rearranged AML (Monocyte - like - MLL) and ALL (KMT2A) (Fig. 6a). To confirm this observation, we subsequently analyzed the transcript levels of LILRB4 in 15 leukemia cell lines with or without MLL-rearrangements from the CCLE database. Leukemia cell lines with presence of MLL-fusion genes exhibited markedly higher LILRB4 expression than those lack MLL-fusion genes, whether LILRB4 expression was detected by RNA-seq (Fig. 6b) or Affymetrix microarray (Supplementary Figure S11a). Accordingly, analysis of three large primary patient datasets (BeatAML, TCGA, and GSE13159) revealed consistently highest LILRB4 expression in MLL-rearranged AML as compared to other cytogenetic/clinicopathologic leukemia entities (Fig. 6c, Supplementary Figure S11b and c). To further confirm the relevance of $L I L R B 4$ expression in MLL-rearranged AML, we collected four MLLrearrangement-related gene signatures from MSigDB and computed ssGSEA scores of these signatures for each sample in the TCGA dataset. Then, we compared the ssGSEA scores computed for high LILRB4expressing samples with those in low LILRB4-expressing samples. We found gene-sets down-regulated in MLL-rearranged AML (MULLIGHAN_MLL_SIGNATURE_1_DN) showed significantly lower ssGSEA scores in LILRB4-high patients than in LILRB4-low patients; whereas for gene-sets up-regulated in MLLrearranged AML (MULLIGHAN_MLL_SIGNATURE_1_UP), the opposite was seen (Fig. 6d). Also, the ssGSEA scores of two MLL-rearranged-governed signatures (ROSS_AML_WITH_MLL_FUSIONS and 
VALK_AML_WITH_11Q23_REARRANGED) were significantly up-regulated in high LILRB4 expressers (Supplementary Figure S11d).

It has been shown that target genes of MLL-fusions were often hypomethylated [44, 45], which is consistent with our previous observations. Also, promoters of these genes were often enriched with transcription activation-associated histone marks (H3K79me2, H3K27ac, and H3K4me3) [32]. To determine whether LILRB4 expression could be directly regulated by MLL-fusion gene, we analyzed a published ChIP-seq dataset (GSE79899) of MLL-fusion proteins, H3K79me2, H3K27ac, and H3K4me3 for MV4-11 (MLL-AF4) and THP-1 (MLL-AF9) cell lines. We found a significant enrichment of MLL-N proteins in the promoter regions of $L I L R B 4$ gene for both cell lines, while punctuated binding peaks of H3K79me2, $\mathrm{H} 3 \mathrm{~K} 27 \mathrm{ac}$, and H3K4me3 were observed in both the promoter and gene body of LILRB4 (Fig. 6e).

Importantly, a similar enrichment of the three epigenetic marks was seen in five other ChIP-seq datasets (H3K79me2 from GSE82116 and GSE71779; H3K27ac from GSE89336 and GSE71776; H3K4me3 from GSE61785 and GSE82116) (Fig. 6f). Overall, these results suggest that MLL fusion proteins may be a direct regulator of LILRB4 expression.

\section{LILRB1 expression correlates with distinct genomic alterations in AML}

We then examined the associations between LILRBs expression and the clinical and genetic characteristics in the TCGA AML cohort. We found an association between LILRBs expression and the French-American-British (FAB) classification of AML: a higher percentage of myelomonocytic or monocytic morphology (M4/M5 subtypes) and a lower percentage of FAB M2/M3 was observed in patients with high LILRBs expression (Fig. 7a). Moreover, high LILRBs expressers were more likely to be > 60 -year-old and less likely to present with favorable cytogenetics (Fig. 7a).

To determine whether LILRB1-LILRB5 correlated with distinct mutational profiles characterized for AML, we identified significantly mutated genes occurred in patients with high and low LILRB1-LILRB4 expression (as stratified by the median expression value of respective genes), using curated mutational data from TCGA. Overall, we found LILRB1 and LILRB5 expression was associated with more mutational events than the other three genes (Fig. 7b). As shown in Fig. 7c, patients with high LILRB1 expression had higher frequency of mutations in U2AF1 (7\% vs $1 \%$ ) and RUNX1 (14\% vs $4 \%$ ), while IDH1 (14\% vs $4 \%$ ) was more frequently mutated in those with low LILRB1 expression. High LILRB5 expression was positively correlated with TP53 mutations and negatively correlated with FLT3 and WT1 mutations. For other three genes, LILRB2 was associated with mutations in IDH1 and STAG2, LILRB3 with WT1, and LILRB4 with RUNX1 (Fig. 7b).

To further explore the association between LILRBs expression and copy number variation (CNV), we performed GISTIC2.0 analysis of TCGA copy number data and assessed copy number alterations between in two patient groups. We focused on $\angle I L R B 1$, as it was consistently dysregulated and showed the greatest mutational events in AML patients. Interestingly, LILRB1-low patients had no somatic copy number alterations (Supplementary Figure S12), whereas LILRB1-high patients had 14 significantly 
deleted regions and four significantly amplified region (FDR $=0.25)$ (Fig. $7 d)$. Interestingly, the majority of genes deleted in LILRB1-high patients were involved in inflammatory responses (including cytokines and genes essential for microbial killing and antigen processing and presentation, see Supplementary data 1 for detail). Also, a number of genes belongs to the cadherin $(C D H)$ and protocadherin $(P C D H)$ family, which often exerts tumor-suppressive functions [46], were significantly deleted. In contrast, LILRB-high AML patients had recurrent amplification at loci essential in AML pathogenesis, including KMT2A and ERG $[47,48]$ (Fig. 7d).

\section{Correlations Between LILRBs and Tumor Immune Infiltrating Cells (TIICs) in AML}

Considering that $L I L R B s$ might play important roles in the TME, we further explored the correlations between $L I L R B$ s and the level of immune cell infiltration in TCGA AML cohort. It is noteworthy that, among the 22 cell types, monocytes had the highest positive correlations with LILRB1-LILRB4 (Fig. 8a), consistent with previous finding that LILRBs were preferentially expressed in monocytic AML $[19,49]$. This monocytic preference was also confirmed in two recently published scRNA-seq datasets of AML (Van Galen AML scRNA, Fig. 8b and FIMM AML scRNA, Supplementary Figure S13a). Interestingly, LILRB4 was exclusively correlated with $\mathrm{M} 2$ macrophages (Fig. 8a), a high immunosuppressive component in the TME. By contrast, LILRBT-LIRB4 were negatively correlated with the infiltrating levels of tumor-suppressive immune cells, such as resting T cells CD4 memory cell, CD8 T cells, memory B cells, plasma cells, and resting NK cells (Fig. 8a). Similar results were found by analyzing the CIBERSORT estimates in the GSE10358 and GSE6891 dataset (Supplementary Figure S13b and c). Importantly, when other methods were used for calculating the relative fractions of TIICs, positive associations between LIRBT-LILRB4 and monocytes were consistently seen, while negative associations between LILRB1LILRB4 and CD8 T cells were proved for most-if not all-methods in all three datasets (Supplementary Figure S13d-f). Further analysis of normal cell populations from the Hemap dataset revealed that LILRBS were highly expressed in myeloid lineage immune cells (monocytes, macrophages, dendric cells, myeloid progenitors, and neutrophils), with consistent low expression in T cells (CD4 + T cells and T/NK cells) (Fig. 8c). Collectively, these findings further confirmed the immunosuppressive roles of LILRBs in cancer TME.

\section{Correlation between LILRBs and immune checkpoints in AML}

Given that immune checkpoints have been proved to be promising therapeutic target for cancer treatment, we therefore evaluated the relationship between LILRBS and a collection of checkpoint genes describe by De Simone et al. [37]. Results from Spearman correlation analyses are given in Supplementary Data 2. As shown in the Circos plots, LILRB1-LILRB3 all showed strong positive correlations with CD86, VISTA, and HAVCR2 (Fig. 8d-f), indicating a possible synergistic effect between these genes. In contrast, no significant correlations were observed between $L I L R B 4 / 5$ and these 
checkpoints (Supplementary Figure S14a and b). These results further highlight LILRBs potentially as major signaling pathways involved in immunosuppression in the AML microenvironment.

\section{The biological significance of LILRBs expression in AML}

We then sought to investigate the biological features associated with $L I L R B s$ in AML. Since the expression of five $L I L R B$ members were highly correlated, a comparison of gene expression profiles of patients with high and low LILRB1 expression (as determined by the median expression value) was performed. Overall, 799 genes (490 up- and 309 downregulated; adjusted $p<0.05$; $\log 2 \mathrm{FC} \leq-1.5$ or $\log 2 \mathrm{FC} \geq 1.5$ ) were differentially expressed in $L I L R B 7^{\text {high }}$ versus $\angle I L R B 7^{\text {low }}$ patients (Fig. 9a and Supplementary Data 3). Among the genes positively correlated with $L I L R B 1$ were, as expected, the other members of the LILRB family (Fig. 9a). Also, genes associated with presence of monocytes/macrophages (CD14,CD68) or M2 macrophage polarization (MSR1, MRC1, CD163) were significantly up-regulated in high LILRB1 expressers (Fig. 9a), in line with our previous findings. Next, we used STRING database to construct a protein-protein interaction (PPI) network of the differentially expressed genes (DEGs), with a confidence score $>0.90$. Genes interacted with LILRB1 and their subnetworks were shown through Cytoscape software (Fig. 9b). We found 12 genes directly interacting with LILRB1: PILRA, TLR8, SIGLEC7, CD300C, FCGR2A, FCGR2B, FCGR3A, CD86, FGR, HCK, IL 10, ITGAX. Among them, CD300C, FCGR2A, FCGR2B, and FCGR3A also had connections with the other four LILRB members (Fig. 9b). GeneMANIA results also revealed that genes of the FCGR and CD300 family were closely correlated with LILRBS. These genes were mainly involved in negative regulation of leukocyte mediated immunity and negative regulation of immune system process (Supplementary Figure S15a).

We then performed $\mathrm{GO}$ analysis using these DEGs and the top 10 significant terms of $\mathrm{BP}, \mathrm{MF}$ and CC enrichment analysis were shown (Fig. 9c). Notably, in terms of BP, immune response-related processes were significantly enriched, such as inflammatory response, immune system process, and immune response. KEGG and Reactome Pathway analyses also revealed immune response pathways, including cytokine - cytokine receptor interaction, cytokine signaling in immune system, innate immune system, antigen processing - cross presentation, and adaptive immune system were mainly enriched (Fig. 11d and Supplementary Figure S15b).

Finally, GSEA was conducted in the $L I L R B 7^{\text {high }}$ and $\angle I L R B 7^{\text {low }}$ cohorts. For the $C 2$ collection of curated gene sets from the MSigDB, the VALK_AML_CLUSTER_5 gene set (96\% of the samples are FAB M4 or M5 subtype) was predominantly enriched in $\angle I L R B 7^{\text {high }}$ group. Also enriched were gene sets of MLL-fusion and NPM1-mutation, two distinct entities often associated with monocytic features of AML (Fig. 10a). For the $C 7$ immunologic collection, the $L I L R B 7^{\text {high }}$ group had principal enrichment in genes up-regulated in monocytes compared to other immune cells (Fig. 10b), and multiple immune activities were enriched in the $L I L R B 7^{\text {high }}$ group for HALLMARK gene sets (Fig. 10c).

\section{Discussion}


The LILRB family members-LILRB1-5-are a group of proteins containing the immune-inhibitory ITIM motifs which negatively regulate immune cell activation [14]. Here, using RNA-seq data of normal tissues from GTEx, FANTOM5, and HPA, we showed that LILRB members were predominantly enriched in the spleen, consistent with their immune modulatory functions. In cancer cell lines, LILRBs showed relatively high expression in cell lines of malignant hematological origin, in line with the selective expression of LILRBs in hematopoietic lineage cells. Indeed, abnormal expression of LILRBs has been documented in various cancers, such as lung cancer [50], hepatocellular carcinoma (HCC) [51], and certain types of subtypes of adenocarcinoma [41]. In this study, based on combined datasets from TCGA and GTEx, we comprehensively analyzed LILRBs expression between tumor and adjacent normal tissue across 28 cancer types (9465 tumor and 7831 normal samples). Our data showed that LILRBs were significantly dysregulated in the majority of tumor types. For LILRB1-4, the most striking difference was seen between AML and its normal counterparts. Although previous study has reported increased expression of LILRB4 in $A M L[19,49]$, this was not seen in two independent validation sets of AML patients, probably due to technical/biological heterogeneity across studies. However, we do note a strong enrichment for LILRBs in the monocytic lineage; this observation was confirmed in HPA pan-cancer cell lines dataset, single-cell transcriptomics of immune cells, immune cell abundances estimated using bulk TCGA samples, and GSEA analysis of monocyte-related gene sets, in agreement with previous reports $[19,20,22,49]$.

Despite being positively correlated with monocytes, LILRB1-4 were negatively correlated with the density of CD8 $+T$ and NK cells, which are considered essential for effective anti-tumor immunity [27]. It has been shown that activated $L I L R B 4$ on monocytic AML cells recruits $S H P-2$ and upregulates $N F K B$, leading to increased $A R G 1$ and $U P A R$ accompanied by a concomitant suppression of $T$ cell activity $[18,19]$. This might provide a potential mechanistic explanation to our observations. It should be noted that bone marrow (BM)-T cells in AML are often functionally impaired [11-13, 52], possibly mediated by malignant monocyte-like cells from AML $[11,19,21,53]$. Further research aimed to unravel the underlying molecular mechanisms is clearly warranted, as this may provide opportunities for identification of new drugs targets and therapeutics that can circumvent the T cell suppression state in AML.

Immunosuppressive factors, such as indoleamine 2,3-dioxygenase 1 (IDO1), CD200, and TIM-3 were reported to be closely associated with a poor outcome in AML [54-56]. In a preliminary analysis, Deng et al. studied the prognostic relevance of several co-stimulating and co-inhibitory receptors in TCGA AML dataset, including LILRB1-LILRB4 [19], but there is limited data and require validation in large datasets. Here, we independently validated the prognostic significances of $L I L R B$ members in five independent datasets. Strikingly, we showed that $L I L R B 1-4$ adversely impacted survival in almost all analyzed datasets. Of interest, we also noticed that LILRB4 was significantly associated with M2 macrophage abundances. This observation raises the possibility that $L I L R B 4$ might contribute to leukemogenesis through M2 macrophages. Our group has recently reported that M2 macrophages fractions were selectively upregulated in AML than other four hematological malignancies and normal controls [10]. Importantly, we also demonstrated a superior predictive performance of the M2 marker CD206 (MRC1) than classical prognosticators in AML. Interestingly, in this study we found that $C D 206$ was significantly up-regulated in high LILRB1 expressers. As CD206+ and/or LILRB4+ monocytes could suppress T-cell 
proliferation and create an immunosuppressive microenvironment in AML $[19,53]$, it could be hypothesized that at least part of the prognostic value of LILRBs could be attributed to the immunesuppressive TME it contributed. Acute monocytic leukemia often harbors mixed-lineage leukemia (MLL) rearrangements, an aggressive phenotype with limited treatment options and poor survival rates, which might also explain the observed result. Indeed, we demonstrated that LILRB4 was aberrantly overexpressed in MLL-rearranged AML and might be a direct target of the MLL fusion proteins.

In a recent pan-hematological-malignancies study, the authors have found that LILRB2 could distinguish lymphoma and leukemia subtypes with high immune infiltration from those harboring lower cytolytic score [27]. We consistently found multiple genes involving in immune activation (including cytokines and genes essential for microbial killing and antigen processing and presentation) were deleted in LILRB1high patients, indicating a delicate balance between immune activation and suppression in the TME.

Indeed, an integrated analysis of transcriptomic and proteomic data has uncovered and ranked LILRBS among the top potential chimeric antigen receptor (CAR) targets in AML [57]. Preliminary evidence from the Gui group also suggests that blocking LILRB4 activation effectively reversed T-cell suppression and inhibited AML cell infiltration [18]. Given that $L I L R B$ s are selectively dysregulated in AML, it is tempting to speculate that AML positive for these proteins might be good candidates for immunotherapy. Future cancer immunotherapy clinical trials will be critical to further validate these findings.

In this study, we provided a comprehensive analysis of the expression patterns and clinical significances of LILRBS across pan-cancers, focusing its role in AML. We also analyzed the association of LILRBS expression with genomic features and tumor immunity in AML. Our data revealed up-regulated expression of $L I L R B s$ in $A M L$ and that higher expression levels of these genes predicted worse outcome. In addition, $L I L R B s$ were associated with an immune-suppressive TME in AML. Overall, these findings suggest important immunological and clinical implications of $L I L R B s$ in $A M L$, which warrants further clinical investigation with immunotherapy specifically targeting AML with LILRBs dysregulations.

\section{Declarations}

\section{Acknowledgements}

Not applicable.

\section{Funding}

This study was supported by National Natural Science foundation of China $(81970118,81900163)$, Medical Innovation Team of Jiangsu Province (CXTDB2017002), Zhenjiang Clinical Research Center of Hematology (SS2018009), Social Development Foundation of Zhenjiang (SH2019065, SH2019067), Scientific Research Project of The Fifth 169 Project of Zhenjiang (21).

\section{Availability of data and materials}


The datasets analyzed in this study are available in the following open access repositories:

GTEx, www.gtexportal.org/

HPA, https://www.proteinatlas.org/

CCLE, https://www.broadinstitute.org/ccle

TCGA, https://portal.gdc.cancer.gov/, http://www.cbioportal.org

GEO, https://www.ncbi.nlm.nih.gov/geo/ (GEO accession numbers: GSE63270, GSE30029, GSE42519, GSE13159, GSE116256, GSE63409, GSE79899, GSE82116, GSE71779, GSE89336, GSE71776, GSE61785, GSE10358, GSE37642, GSE106291, and GSE71014)

FIMM AML scRNA data, https://www.synapse.org (doi: 10.7303/syn21991014)

DiseaseMeth, http://bio-bigdata.hrbmu.edu.cn/diseasemeth/analyze.html

TIMER 2.0, http://timer.comp-genomics.org/

\section{Author contributions}

JQ, JL, and Z-WM conceived and designed the study; Z-JX, X-LZ, YJ, S-SW, and YG collected and assembled data; Z-JX, J-CM, X-MW, and J-YL performed data analysis; Z-JX drafted the manuscript; JQ, $\mathrm{JL}$, and Z-WM participated in study supervision and commented on the manuscript. All authors read and approved the final manuscript.

\section{Ethics approval and consent to participate}

Not applicable.

\section{Consent for publication}

Not applicable.

\section{Competing interests}

The authors declare that they have no competing interests.

\section{References}

1. Löwenberg B, Downing JR, Burnett A: Acute myeloid leukemia. N Engl J Med 1999, 341(14):10511062.

2. Byrd JC, Mrózek K, Dodge RK, Carroll AJ, Edwards CG, Arthur DC et al: Pretreatment cytogenetic abnormalities are predictive of induction success, cumulative incidence of relapse, and overall 
survival in adult patients with de novo acute myeloid leukemia: results from Cancer and Leukemia Group B (CALGB 8461). Blood 2002, 100(13):4325-4336.

3. Marcucci G, Mrózek K, Bloomfield CD: Molecular heterogeneity and prognostic biomarkers in adults with acute myeloid leukemia and normal cytogenetics. Curr Opin Hematol 2005, 12(1):68-75.

4. Döhner H, Estey EH, Amadori S, Appelbaum FR, Büchner T, Burnett AK et al: Diagnosis and management of acute myeloid leukemia in adults: recommendations from an international expert panel, on behalf of the European LeukemiaNet. Blood 2010, 115(3):453-474.

5. DiNardo CD, Pratz KW, Letai A, Jonas BA, Wei AH, Thirman M et al: Safety and preliminary efficacy of venetoclax with decitabine or azacitidine in elderly patients with previously untreated acute myeloid leukaemia: a non-randomised, open-label, phase 1b study. Lancet Oncol 2018, 19(2):216-228.

6. DiNardo CD, Maiti A, Rausch CR, Pemmaraju N, Naqvi K, Daver NG et al: 10-day decitabine with venetoclax for newly diagnosed intensive chemotherapy ineligible, and relapsed or refractory acute myeloid leukaemia: a single-centre, phase 2 trial. Lancet Haematol 2020, 7(10):e724-e736.

7. Topalian SL, Hodi FS, Brahmer JR, Gettinger SN, Smith DC, McDermott DF et al: Safety, activity, and immune correlates of anti-PD-1 antibody in cancer. N Engl J Med 2012, 366(26):2443-2454.

8. Brahmer JR, Tykodi SS, Chow LQ, Hwu WJ, Topalian SL, Hwu P et al: Safety and activity of anti-PDL1 antibody in patients with advanced cancer. N Engl J Med 2012, 366(26):2455-2465.

9. Lichtenegger FS, Krupka C, Haubner S, Köhnke T, Subklewe M: Recent developments in immunotherapy of acute myeloid leukemia. J Hematol Oncol 2017, 10(1):142.

10. Xu ZJ, Gu Y, Wang CZ, Jin Y, Wen XM, Ma JC et al: The M2 macrophage marker CD206: a novel prognostic indicator for acute myeloid leukemia. Oncoimmunology 2020, 9(1):1683347.

11. van Galen P, Hovestadt V, Wadsworth li MH, Hughes TK, Griffin GK, Battaglia S et al: Single-Cell RNASeq Reveals AML Hierarchies Relevant to Disease Progression and Immunity. Cel/ 2019, 176(6):12651281.e1224.

12. Lamble AJ, Kosaka Y, Laderas T, Maffit A, Kaempf A, Brady LK et al: Reversible suppression of T cell function in the bone marrow microenvironment of acute myeloid leukemia. Proc Natl Acad Sci USA 2020, 117(25):14331-14341.

13. Noviello M, Manfredi F, Ruggiero E, Perini T, Oliveira G, Cortesi F et al: Bone marrow central memory and memory stem T-cell exhaustion in AML patients relapsing after HSCT. Nat Commun 2019, 10(1):1065.

14. Kang X, Kim J, Deng M, John S, Chen H, Wu G et al: Inhibitory leukocyte immunoglobulin-like receptors: Immune checkpoint proteins and tumor sustaining factors. Cell Cycle 2016, 15(1):25-40.

15. Banchereau J, Zurawski S, Thompson-Snipes L, Blanck JP, Clayton S, Munk A et al: Immunoglobulinlike transcript receptors on human dermal CD14+ dendritic cells act as a CD8-antagonist to control cytotoxic T cell priming. Proc Natl Acad Sci U S A 2012, 109(46):18885-18890.

16. Baudhuin J, Migraine J, Faivre V, Loumagne L, Lukaszewicz AC, Payen D et al: Exocytosis acts as a modulator of the ILT4-mediated inhibition of neutrophil functions. Proc Natl Acad Sci U S A 2013, 110(44):17957-17962.

Page $18 / 31$ 
17. van der Touw W, Chen HM, Pan PY, Chen SH: LILRB receptor-mediated regulation of myeloid cell maturation and function. Cancer Immunol Immunother 2017, 66(8):1079-1087.

18. Gui X, Deng M, Song H, Chen Y, Xie J, Li Z et al: Disrupting LILRB4/APOE Interaction by an Efficacious Humanized Antibody Reverses T-cell Suppression and Blocks AML Development. Cancer Immunol Res 2019, 7(8):1244-1257.

19. Deng M, Gui X, Kim J, Xie L, Chen W, Li Z et al: LILRB4 signalling in leukaemia cells mediates $T$ cell suppression and tumour infiltration. Nature 2018, 562(7728):605-609.

20. Barkal AA, Weiskopf K, Kao KS, Gordon SR, Rosental B, Yiu YY et al: Engagement of MHC class I by the inhibitory receptor LILRB1 suppresses macrophages and is a target of cancer immunotherapy. Nat Immuno/ 2018, 19(1):76-84.

21. Knaus HA, Berglund S, Hackl H, Montiel-Esparza R, Levis MJ, Karp JE et al: Acute Myeloid Leukemia (AML) Blasts Influence the Gene Expression Signature and Co-Signaling Receptor Expression of CD8+ T Cells. Blood 2016, 128(22):1700-1700.

22. Zheng J, Umikawa M, Cui C, Li J, Chen X, Zhang C et al: Inhibitory receptors bind ANGPTLs and support blood stem cells and leukaemia development. Nature 2012, 485(7400):656-660.

23. Consortium G: The Genotype-Tissue Expression (GTEx) project. Nat Genet 2013, 45(6):580-585.

24. Pontén F, Jirström K, Uhlen M: The Human Protein Atlas--a tool for pathology. J Pathol 2008, 216(4):387-393.

25. Barretina J, Caponigro G, Stransky N, Venkatesan K, Margolin AA, Kim S et al: The Cancer Cell Line Encyclopedia enables predictive modelling of anticancer drug sensitivity. Nature 2012, 483(7391):603-607.

26. Ritchie ME, Phipson B, Wu D, Hu Y, Law CW, Shi W et al: limma powers differential expression analyses for RNA-sequencing and microarray studies. Nucleic Acids Res 2015, 43(7):e47.

27. Dufva $O$, Pölönen P, Brück O, Keränen MAl, Klievink J, Mehtonen J et al: Immunogenomic Landscape of Hematological Malignancies. Cancer Cel/ 2020, 38(3):380-399.e313.

28. Tyner JW, Tognon CE, Bottomly D, Wilmot B, Kurtz SE, Savage SL et al: Functional genomic landscape of acute myeloid leukaemia. Nature 2018, 562(7728):526-531.

29. Mayakonda A, Lin DC, Assenov Y, Plass C, Koeffler HP: Maftools: efficient and comprehensive analysis of somatic variants in cancer. Genome Res 2018, 28(11):1747-1756.

30. Mermel CH, Schumacher SE, Hill B, Meyerson ML, Beroukhim R, Getz G: GISTIC2.0 facilitates sensitive and confident localization of the targets of focal somatic copy-number alteration in human cancers. Genome Biol 2011, 12(4):R41.

31. Liu CJ, Hu FF, Xia MX, Han L, Zhang Q, Guo AY: GSCALite: a web server for gene set cancer analysis. Bioinformatics 2018, 34(21):3771-3772.

32. Prange KHM, Mandoli A, Kuznetsova T, Wang SY, Sotoca AM, Marneth AE et al: MLL-AF9 and MLLAF4 oncofusion proteins bind a distinct enhancer repertoire and target the RUNX1 program in 11q23 acute myeloid leukemia. Oncogene 2017, 36(23):3346-3356. 
33. Li T, Fu J, Zeng Z, Cohen D, Li J, Chen Q et al: TIMER2.0 for analysis of tumor-infiltrating immune cells. Nucleic Acids Res 2020, 48(W1):W509-W514.

34. Camp RL, Dolled-Filhart M, Rimm DL: X-tile: a new bio-informatics tool for biomarker assessment and outcome-based cut-point optimization. Clin Cancer Res 2004, 10(21):7252-7259.

35. Tamborero D, Rubio-Perez C, Muiños F, Sabarinathan R, Piulats JM, Muntasell A et al: A Pan-cancer Landscape of Interactions between Solid Tumors and Infiltrating Immune Cell Populations. Clin Cancer Res 2018, 24(15):3717-3728.

36. Sturm G, Finotello F, Petitprez F, Zhang JD, Baumbach J, Fridman WH et al: Comprehensive evaluation of transcriptome-based cell-type quantification methods for immuno-oncology. Bioinformatics 2019, 35(14):i436-i445.

37. De Simone M, Arrigoni A, Rossetti G, Gruarin P, Ranzani V, Politano C et al: Transcriptional Landscape of Human Tissue Lymphocytes Unveils Uniqueness of Tumor-Infiltrating T Regulatory Cells. Immunity 2016, 45(5):1135-1147.

38. Maag JLV: gganatogram: An R package for modular visualisation of anatograms and tissues based on ggplot2. F1000Res 2018, 7:1576.

39. The Genotype-Tissue Expression (GTEx) project. Nat Genet 2013, 45(6):580-585.

40. Sun Y, Liu J, Gao P, Wang Y, Liu C: Expression of Ig-like transcript 4 inhibitory receptor in human nonsmall cell lung cancer. Chest 2008, 134(4):783-788.

41. Cheng J, Gao X, Zhang X, Guo H, Chen S, Gou X: Leukocyte immunoglobulin-like receptor subfamily $B$ member 1 potentially acts as a diagnostic and prognostic target in certain subtypes of adenocarcinoma. Med Hypotheses 2020, 144:109863.

42. Rapin N, Bagger FO, Jendholm J, Mora-Jensen H, Krogh A, Kohlmann A et al: Comparing cancer vs normal gene expression profiles identifies new disease entities and common transcriptional programs in AML patients. Blood 2014, 123(6):894-904.

43. Su R, Dong L, Li Y, Gao M, Han L, Wunderlich M et al: Targeting FTO Suppresses Cancer Stem Cell Maintenance and Immune Evasion. Cancer Cell 2020, 38(1):79-96.e11.

44. Hurtz C, Chan LN, Geng H, Ballabio E, Xiao G, Deb G et al: Rationale for targeting BCL6 in MLLrearranged acute lymphoblastic leukemia. Genes Dev 2019, 33(17-18):1265-1279.

45. Akalin A, Garrett-Bakelman FE, Kormaksson M, Busuttil J, Zhang L, Khrebtukova I et al: Base-pair resolution DNA methylation sequencing reveals profoundly divergent epigenetic landscapes in acute myeloid leukemia. PLoS Genet 2012, 8(6):e1002781.

46. Xu ZJ, Ma JC, Zhou JD, Wen XM, Yao DM, Zhang W et al: Reduced protocadherin17 expression in leukemia stem cells: the clinical and biological effect in acute myeloid leukemia. $J$ Trans/ Med 2019, 17(1):102.

47. Sakhdari A, Tang Z, Ok CY, Bueso-Ramos CE, Medeiros LJ, Huh YO: Homogeneously staining region (hsr) on chromosome 11 is highly specific for KMT2A amplification in acute myeloid leukemia (AML) and myelodysplastic syndrome (MDS). Cancer Genet 2019, 238:18-22. 
48. Peterson JF, Sukov WR, Pitel BA, Smoley SA, Pearce KE, Meyer RG et al: Acute leukemias harboring KMT2A/MLLT10 fusion: a 10-year experience from a single genomics laboratory. Genes Chromosomes Cancer 2019, 58(8):567-577.

49. John S, Chen H, Deng M, Gui X, Wu G, Chen W et al: A Novel Anti-LILRB4 CAR-T Cell for the Treatment of Monocytic AML. Mol Ther 2018, 26(10):2487-2495.

50. Liu X, Yu X, Xie J, Zhan M, Yu Z, Xie L et al: ANGPTL2/LILRB2 signaling promotes the propagation of lung cancer cells. Oncotarget 2015, 6(25):21004-21015.

51. Cheng J, Luan J, Chen P, Kuang X, Jiang P, Zhang R et al: Immunosuppressive receptor LILRB1 acts as a potential regulator in hepatocellular carcinoma by integrating with SHP1. Cancer Biomark 2020, 28(3):309-319.

52. Uhl FM, Chen S, O'Sullivan D, Edwards-Hicks J, Richter G, Haring E et al: Metabolic reprogramming of donor T cells enhances graft-versus-leukemia effects in mice and humans. Sci Transl Med 2020, 12(567).

53. Mussai F, De Santo C, Abu-Dayyeh I, Booth S, Quek L, McEwen-Smith RM et al: Acute myeloid leukemia creates an arginase-dependent immunosuppressive microenvironment. Blood 2013, 122(5):749-758.

54. Curti A, Aluigi M, Pandolfi S, Ferri E, Isidori A, Salvestrini V et al: Acute myeloid leukemia cells constitutively express the immunoregulatory enzyme indoleamine 2,3-dioxygenase. Leukemia 2007, 21(2):353-355.

55. Tonks A, Hills R, White P, Rosie B, Mills KI, Burnett AK et al: CD200 as a prognostic factor in acute myeloid leukaemia. Leukemia 2007, 21(3):566-568.

56. Li C, Chen X, Yu X, Zhu Y, Ma C, Xia R et al: Tim-3 is highly expressed in T cells in acute myeloid leukemia and associated with clinicopathological prognostic stratification. Int J Clin Exp Pathol 2014, 7(10):6880-6888.

57. Perna F, Berman SH, Soni RK, Mansilla-Soto J, Eyquem J, Hamieh M et al: Integrating Proteomics and Transcriptomics for Systematic Combinatorial Chimeric Antigen Receptor Therapy of AML. Cancer Cell 2017, 32(4):506-519.e505.

\section{Figures}


A

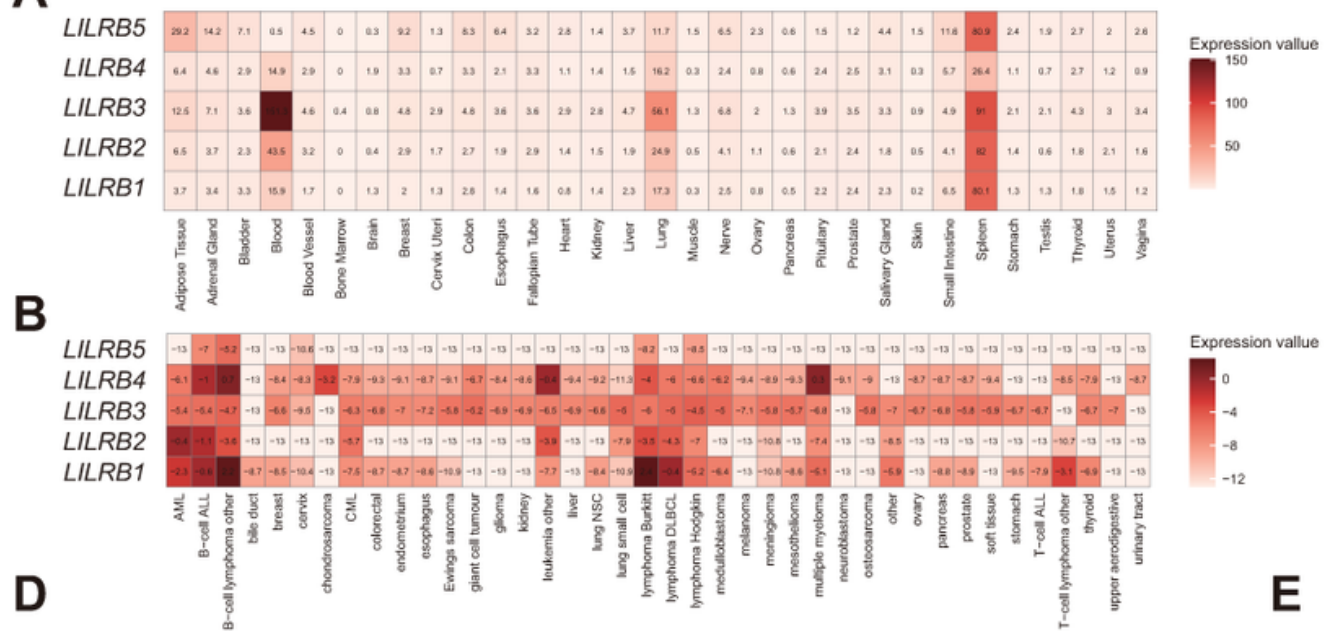

C

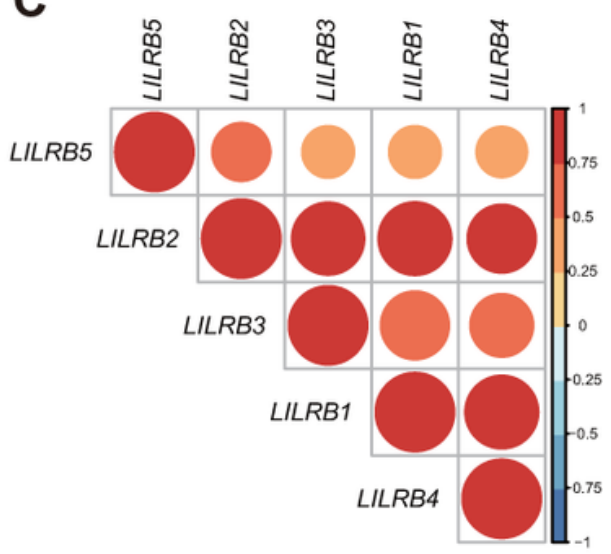

E

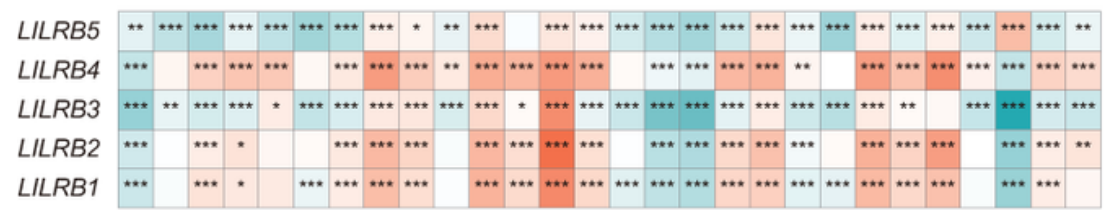

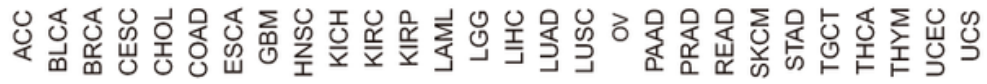

$\mathbf{F}$
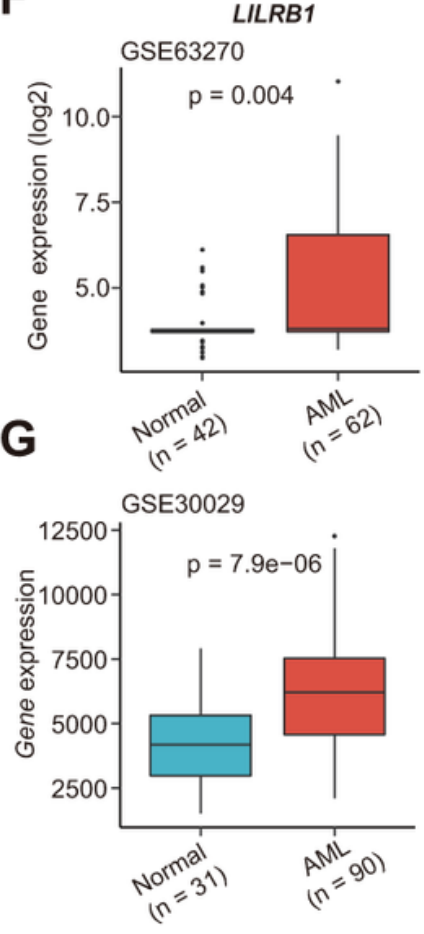

LILRB2
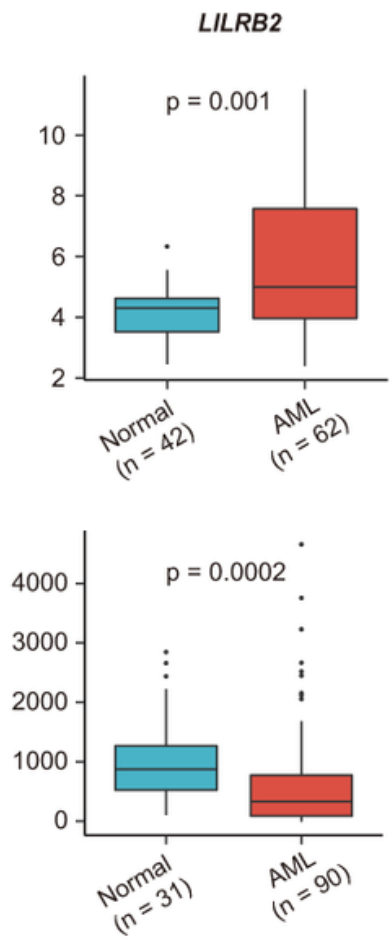

$\log 2$ (FC) $\quad$ LILRB5

2.5 LILRB4 LILRB3 0.0 LILRB2 -2.5 LILRB1 $-5.0 \quad 20$
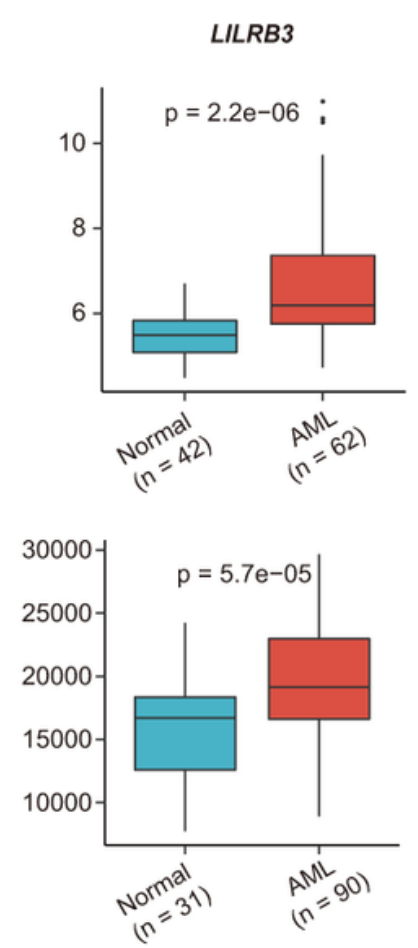

LILRB4
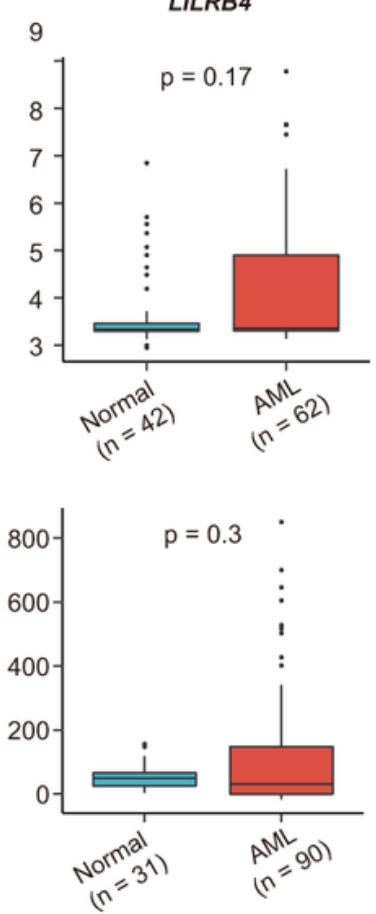

\section{Figure 1}

Expression patterns of LILRBs in normal tissues, cancer cell lines, and primary tumor samples. (a) Heatmap showing mRNA expression levels of LILRBs in normal tissues from the Genotype-Tissue Expression (GTEx) database. (b) Heatmap showing mRNA expression levels of LILRBs in various tumor cell lines from the Cancer Cell Line Encyclopedia (CCLE) database. (c) Correlation matrix of the expression of LILRB1-LILRB5 in the TCGA pan-cancer dataset. (d) Heatmap of differential expression profiles of LILRBs between tumor and normal samples, combining data from TCGA and GTEx databases. 
The color depicts the log2-transformed fold change (Log2FC) between tumor and normal tissues. * $\mathrm{P}<$ $0.05 ; * \star P<0.01 ; * \star * P<0.001$. (e) Bar plot showing genes significantly upregulated and downregulated $(P$ $<0.05$ ) across different cancer types. Red, up-regulated expression; blue, down-regulated expression. ( $f$ and g) Box plots showing expression levels of LILRB1-4 in normal controls and AML in the GSE63270 (f) and GSE30029 (g) datasets.

a

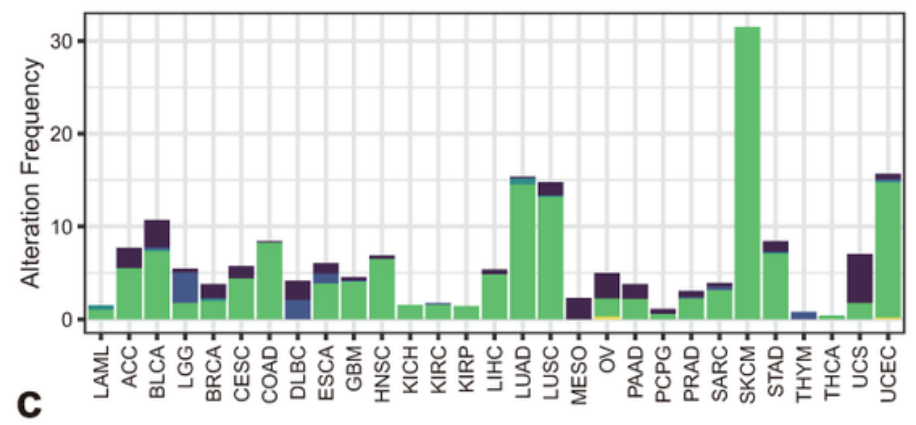

b

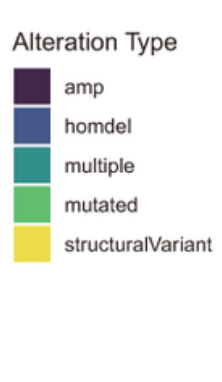

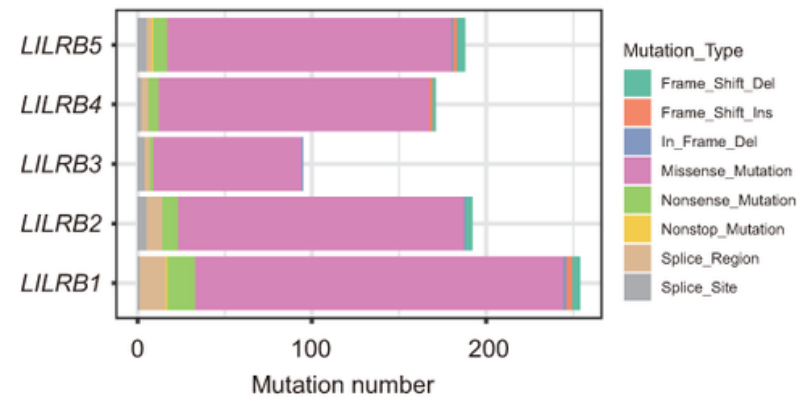

Alteration Frequency

\begin{tabular}{|c|c|c|c|c|c|c|c|c|c|c|c|c|c|c|c|c|c|c|c|c|c|c|c|c|c|c|}
\hline 35 & 0 & 1 & & 2 & & 1 & 3 & .1 & 0.7 & 1 & 5 & & 0 & .4 & 2.7 & 3.9 & 0.2 & 0.5 & 0.6 & .4 & 6 & 7.2 & 5 & 0 & 1.8 & \\
\hline & 0 & & & & & & & & & & & & & & & & & & & .2 & 4 & & & & & \\
\hline$I L F$ & & 0 & & & & & & & & & & & & & & & & & & & 4 & & & & & \\
\hline 20 & 0.5 & & & & & & & & & & & & & 0 & & & & & & & 0.4 & 3 & & & 0 & \\
\hline & 0 & .2 & 1.1 & & & 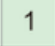 & & . & & & 0 & & & & & 3.5 & & & & & 0 & & & & & \\
\hline & $\sum_{5}^{+}$ & $\begin{array}{l}U \\
\bigcup\end{array}$ & త্త & $\begin{array}{l}0 \\
0 \\
\end{array}$ & $\begin{array}{l}\overleftarrow{\Upsilon} \\
\frac{\Upsilon}{\infty}\end{array}$ & $\begin{array}{l}\text { 凹 } \\
\ddot{W}\end{array}$ & 通 & $\begin{array}{l}\overleftarrow{J} \\
\mho \\
\amalg\end{array}$ & 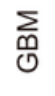 & $\begin{array}{l}\text { U } \\
\text { 足 }\end{array}$ & $\frac{I}{U}$ & $\stackrel{\mathcal{u}}{\underline{\underline{x}}}$ & $\stackrel{\underline{\alpha}}{\underline{\underline{\underline{\alpha}}}}$ & 呈 & 怘 & U & ठ & $\frac{0}{4}$ & $\begin{array}{l}0 \\
0 \\
0\end{array}$ & $\frac{0}{\frac{\alpha}{\alpha}}$ & 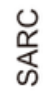 & v & $\frac{\partial}{2}$ & 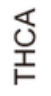 & లુ & \\
\hline
\end{tabular}

10.0

7.5

5.0

2.5

0.0

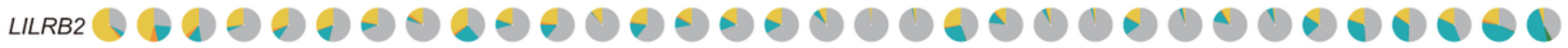

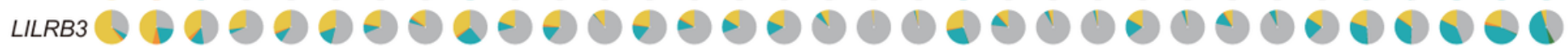

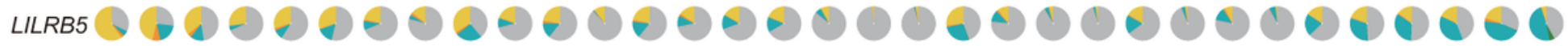

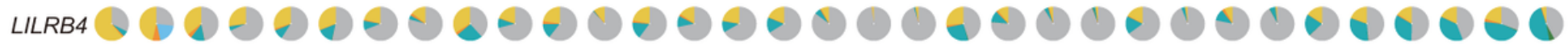

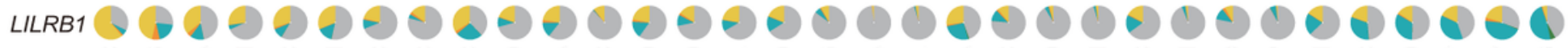

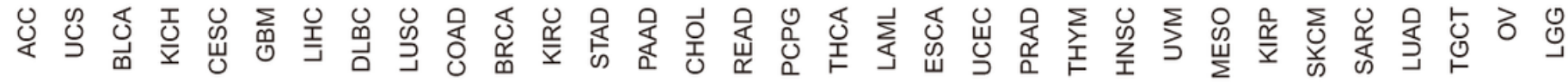
1 Hete Amp HomoAmp Hete Del $\square$ Homo Del

\section{Figure 2}

Landscape of genetic alterations of LILRBs in tumors. (a) Genetic alteration frequencies of LILRBs across different tumors from TCGA. (b) Bar plot showing the percentages of various mutation types for five LILRB genes. (c) Heat map showing mutation frequencies of LILRBs across different cancer types. Numbers on the cells represent mutation percentages. (d) Pie plots showing the percentages of various copy number alteration (CNA) types for five LILRB genes. 


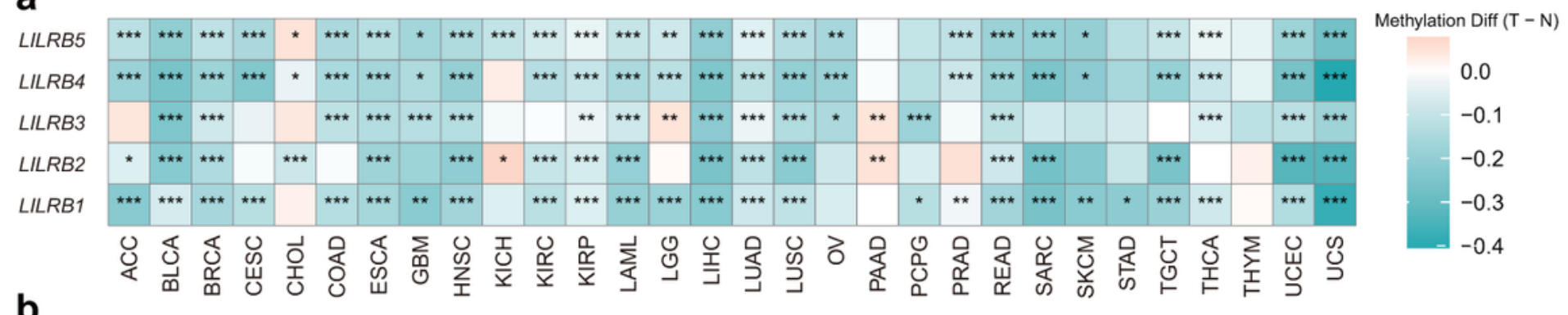

b

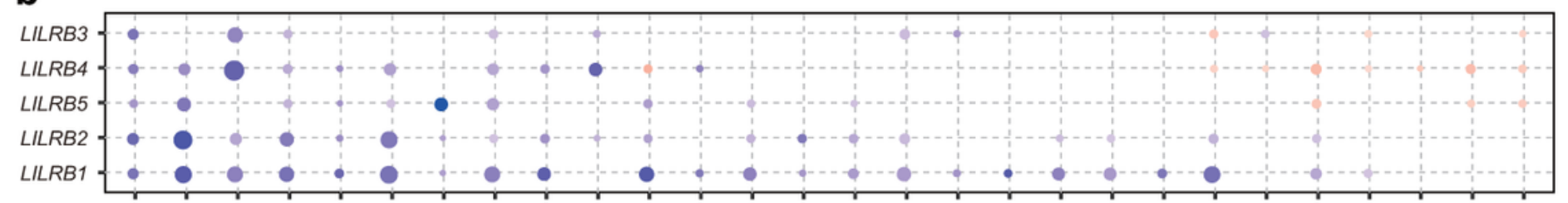

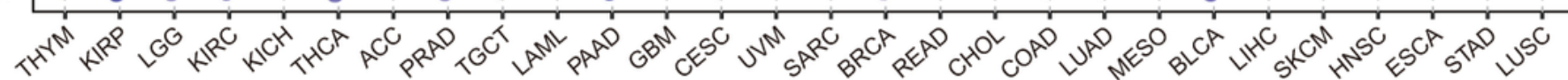

Spearman Correlation Coefficient
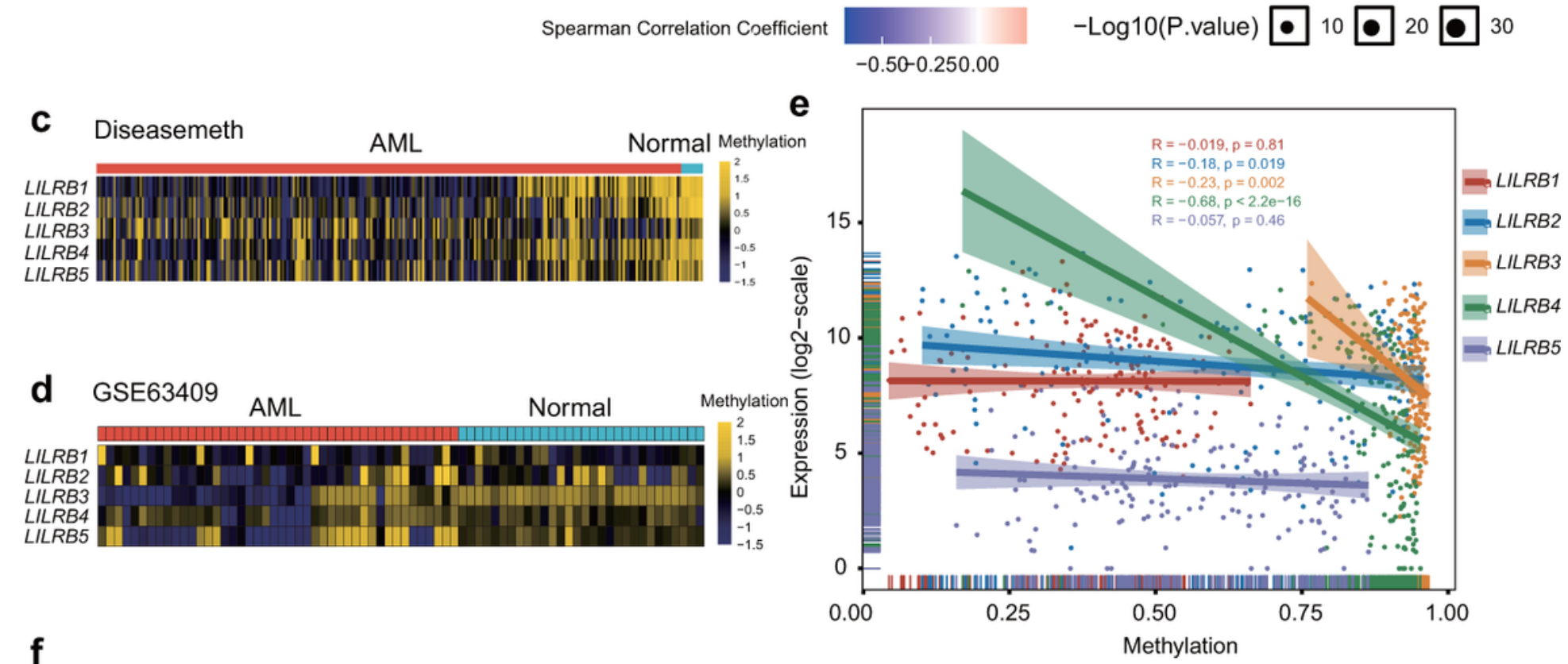

f

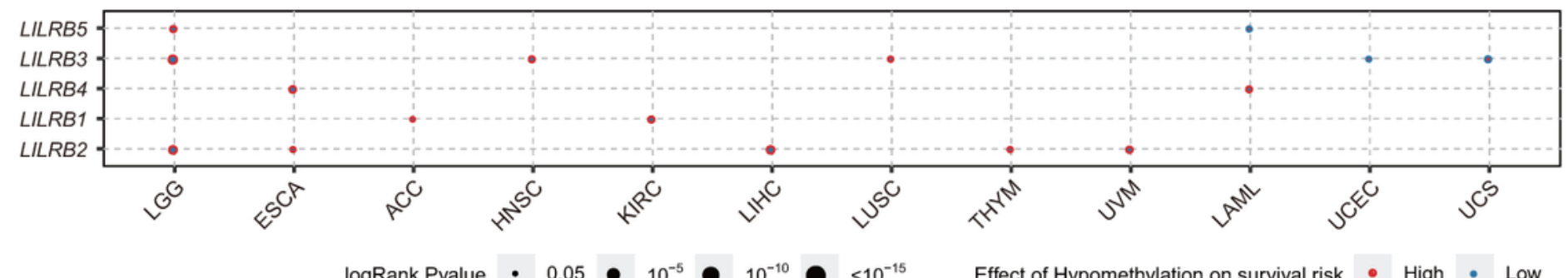

Figure 3

DNA methylation aberration of LILRBs in tumors. (a) Heatmap of differential methylation profiles of LILRBs between tumor and normal samples, using data from the Diseasemeth database. The color depicts methylation differences between tumor $(T)$ and normal $(N)$ tissues. ${ }^{*} P<0.05$; ${ }^{\star * P}<0.01$; $* \star * P<$ 0.001. (b) Correlation between methylation and mRNA expression of LILRBs analyzed via the GSCALite platform. Blue dots indicate negative correlation and red indicate positive correlation. The size of the 
point represents the statistical significance. ( $c$ and d) Heatmap showing methylation of LILRB genes in the Diseasemeth dataset (AML, $n=271$; Normal, $n=10)(c)$ and the GSE63409 dataset $(A M L, n=44$; Normal, $n=30)(d)$. (e) Scatterplot showing the correlation between mRNA expression and DNA methylation levels of LILRBs in the TCGA AML dataset. Spearman correlation coefficients and p-values are indicated. The linear models describing the correlations are depicted as straight lines. The marginal rugs drawn on the axis of the scatter plots were used to show the distributions of two variables. (f) Correlation between LILRBs methylation and survival across cancers analyzed via the GSCALite platform. Red dots indicate a higher risk conveyed by hypomethylation and blue indicate lower risk. The size of the point represents the statistical significance.

a

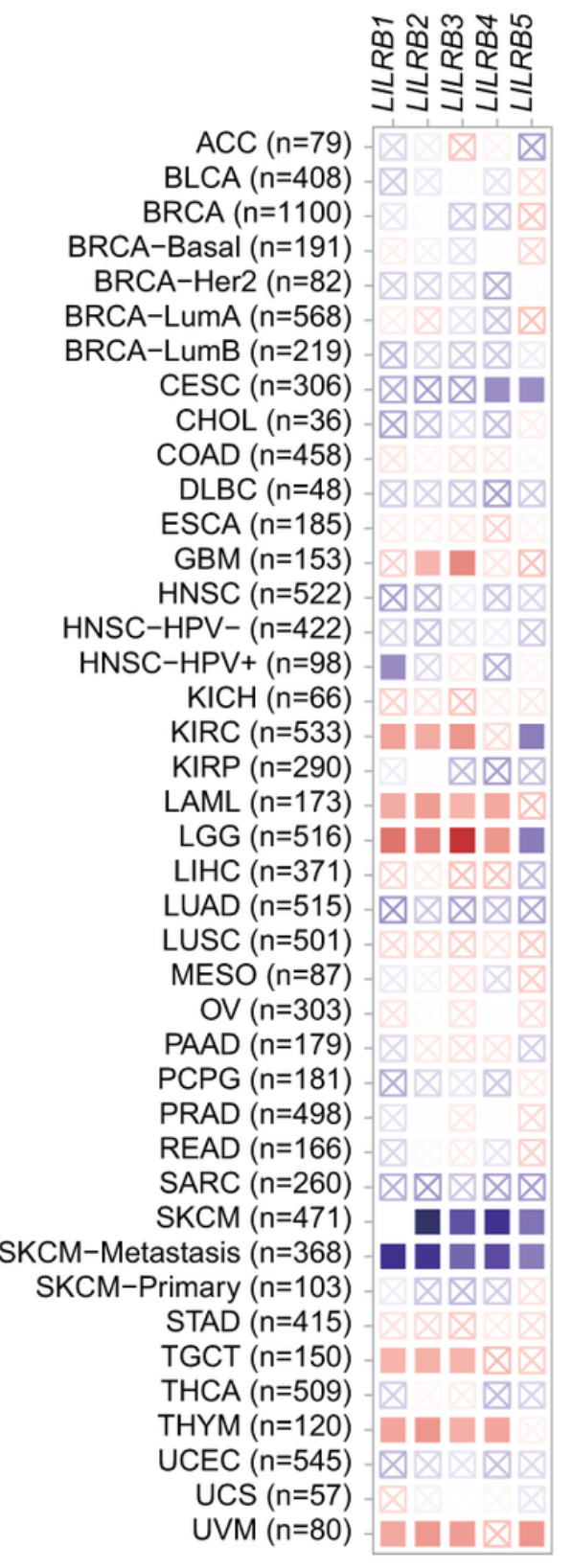

b
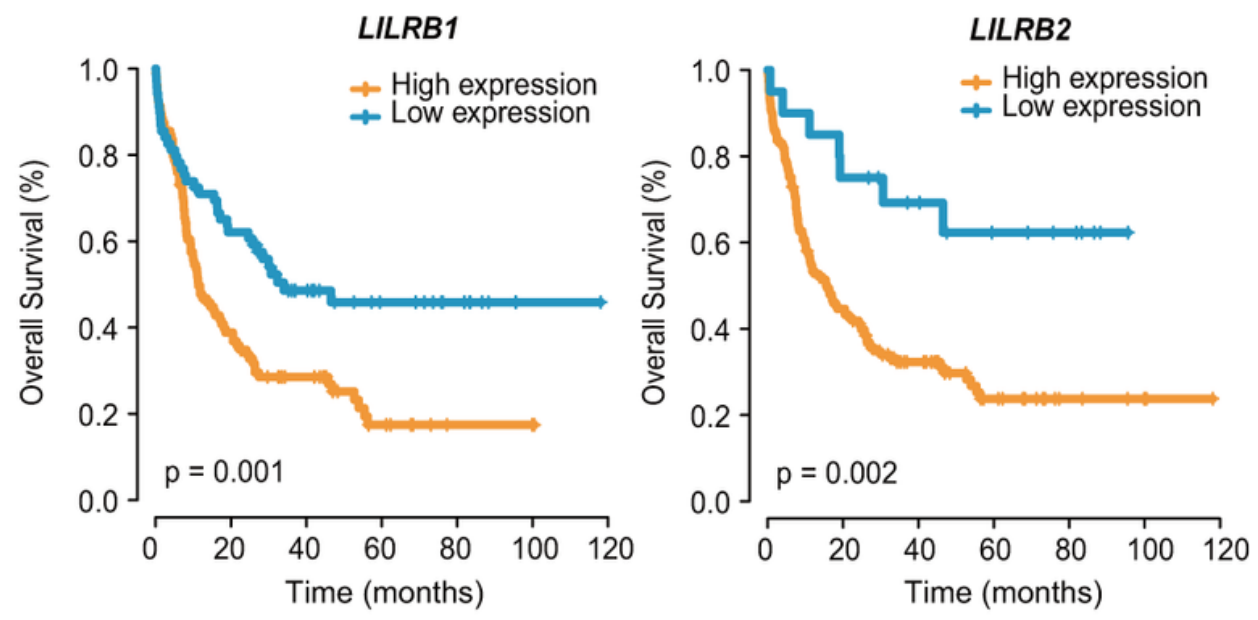

LILRB3
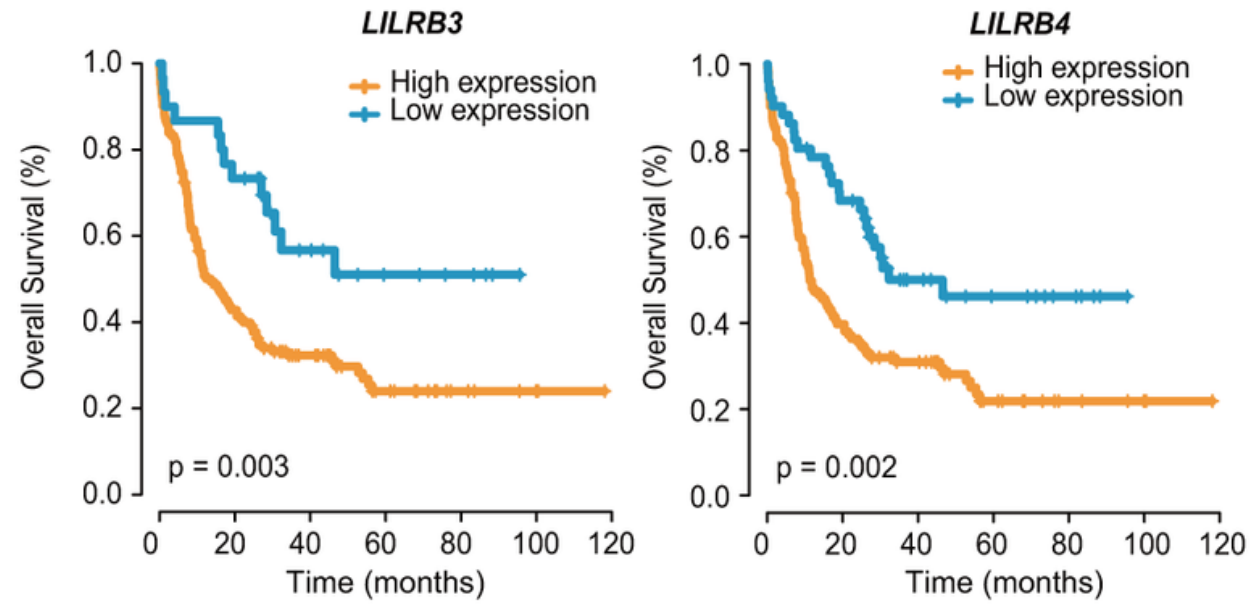

$\otimes p>0.05$

口 $\mathrm{p} \leq 0.05$

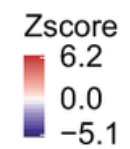

Figure 4 
The prognostic impacts of LILRBs in cancers. (a) Association between LILRBs expression and patient prognosis across 33 cancer types as determined by the TIMER2.0 database. (b) Kaplan-Meier curves representing OS of AML patients from the TCGA cohort based on the expression of indicated LILRB members (LILRB1-LILRB4).

a LILRB1

GSE10358 AML

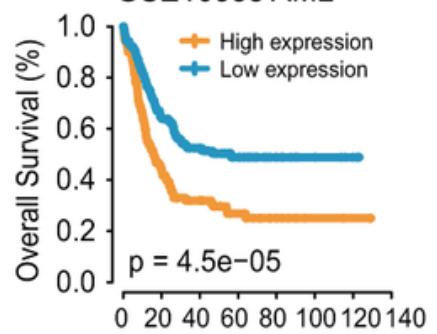

b GSE37642_U133AAML

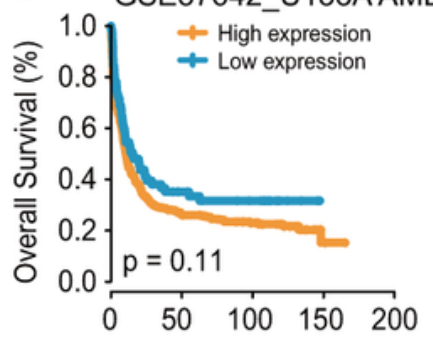

C GSE37642_U133_Plus_2 AML

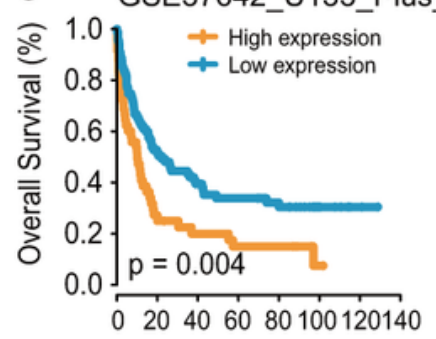

d GSE106291 AML

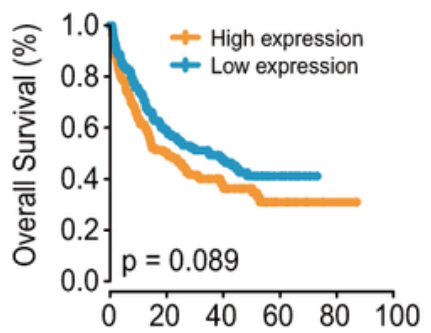

e GSE71014 CN-AML

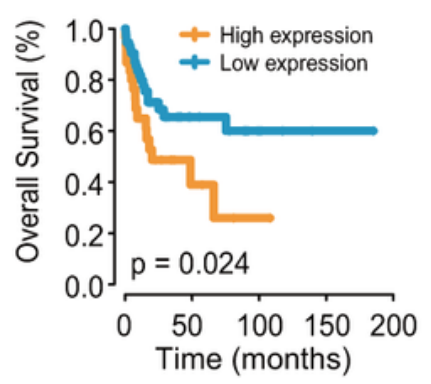

LILRB2
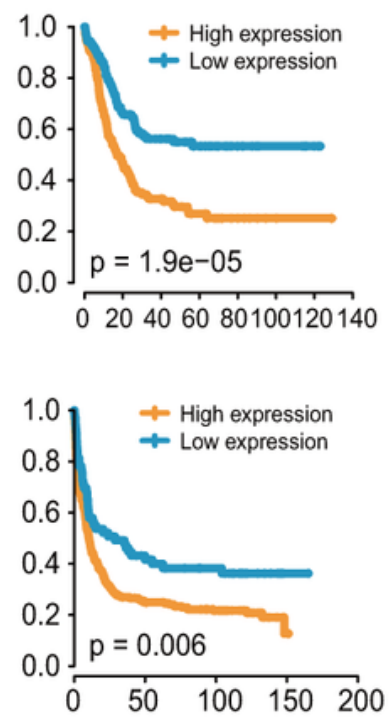

LILRB3
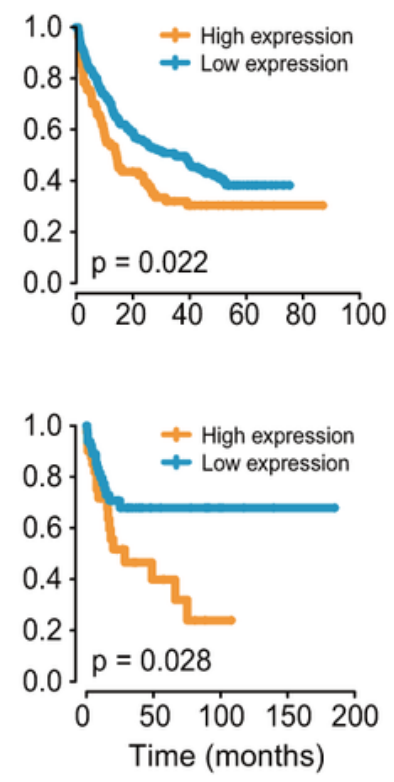
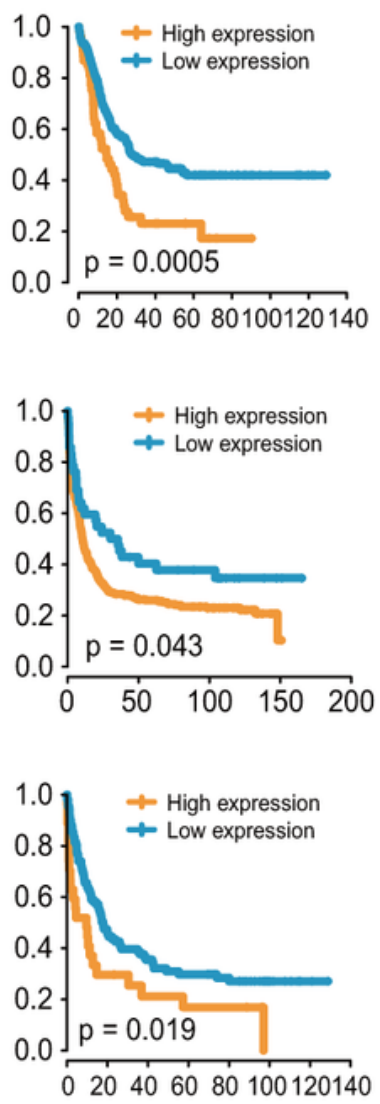

LILRB4
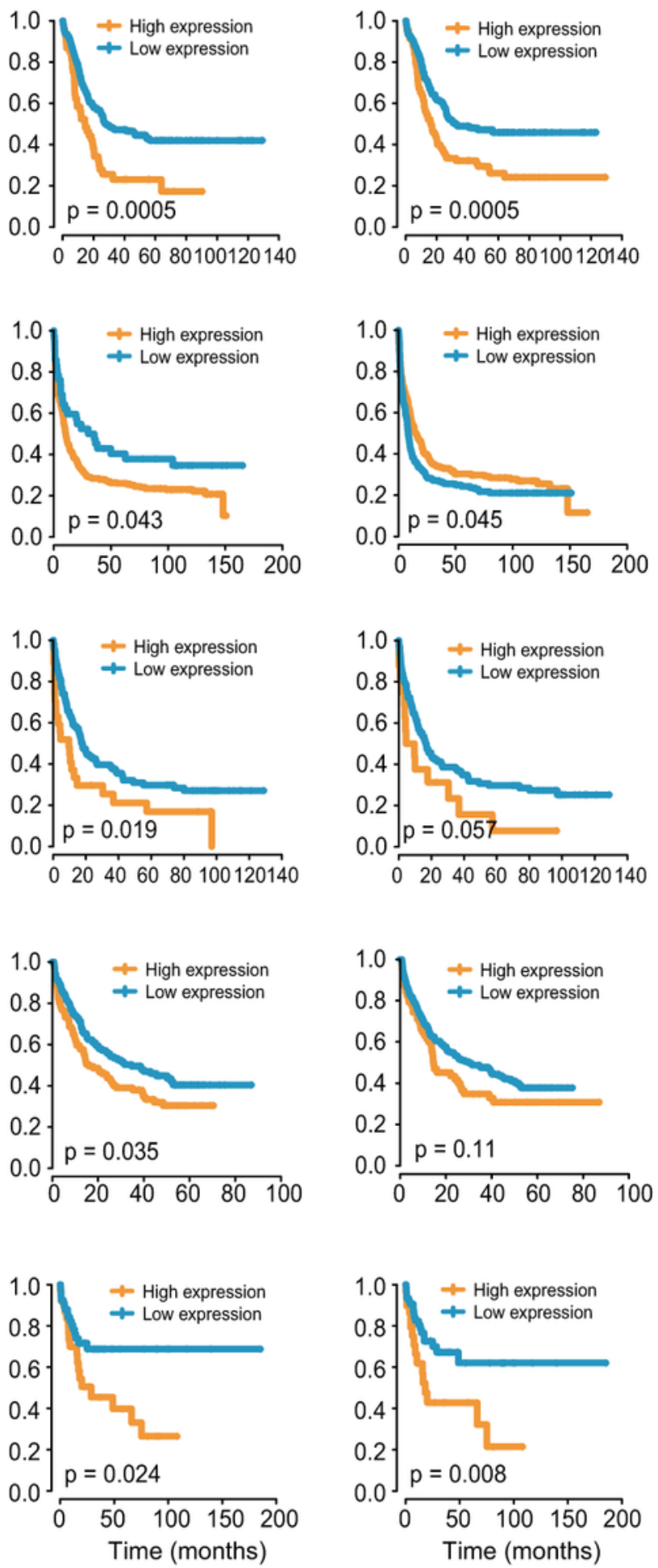

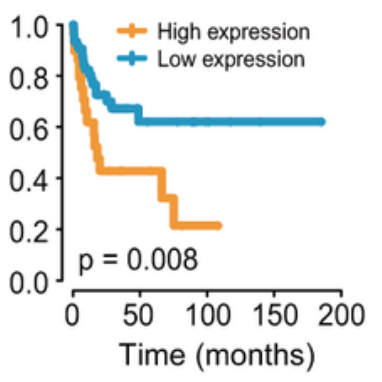

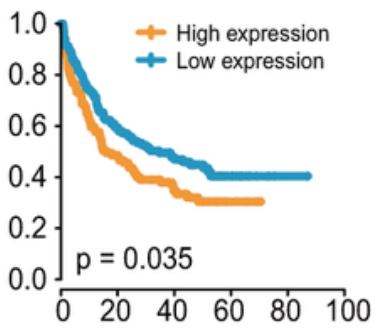

Figure 5 
Independent validation of the prognostic significance of LILRBs in five GEO datasets. (a-e) Kaplan-Meier curves representing OS of five AML cohorts from GEO (GSE10358, $n=304$; GSE37642 [U133A], $n=422$; GSE37642 [U133plus2], $n=140 ;$ GSE106291, $n=250 ;$ GSE71014, $n=104$ ) based on the expression of indicated LILRB members (LILRB1-LILRB4).

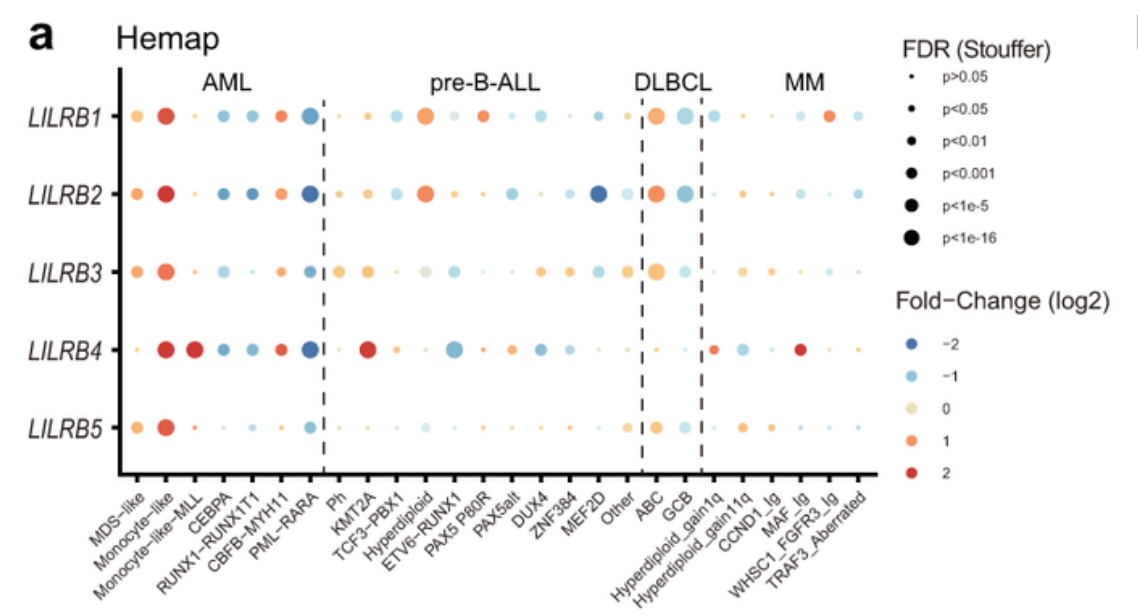

b CCLE RNAseq

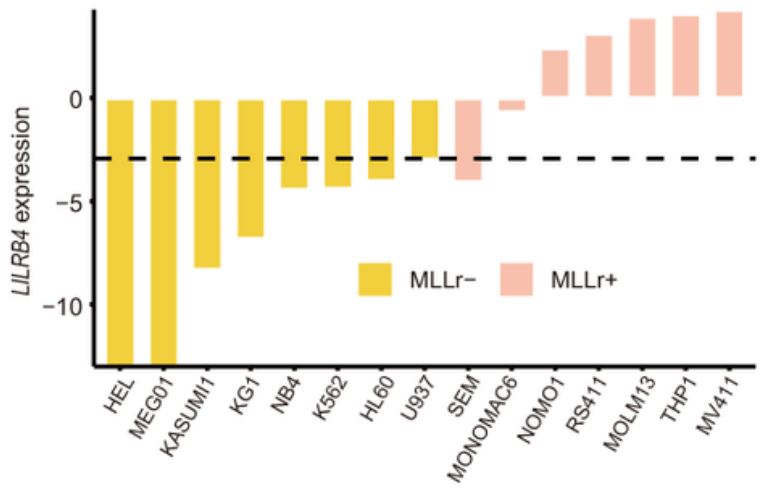

C BeatAML

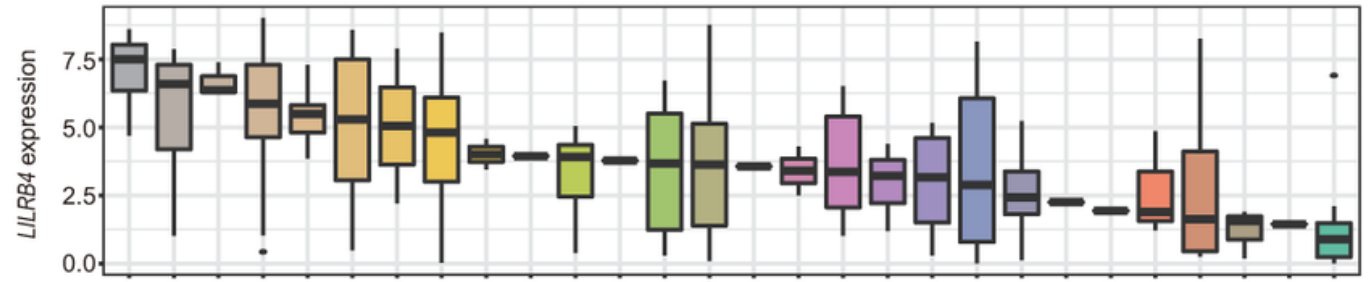

d MULLIGHAN_MLL_SIGNATURE_1_DN

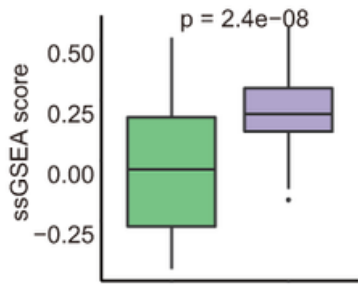

MULLIGHAN_MLL_SIGNATURE_1_UP

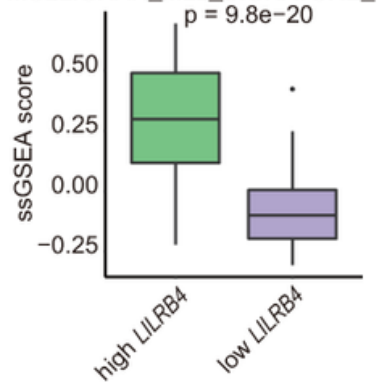

e

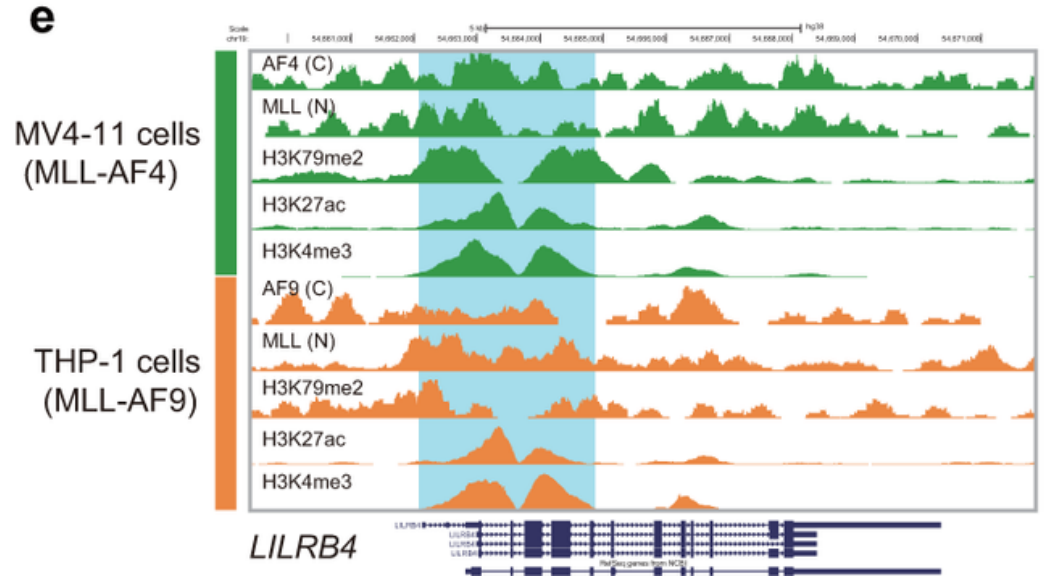

$\mathbf{f}$

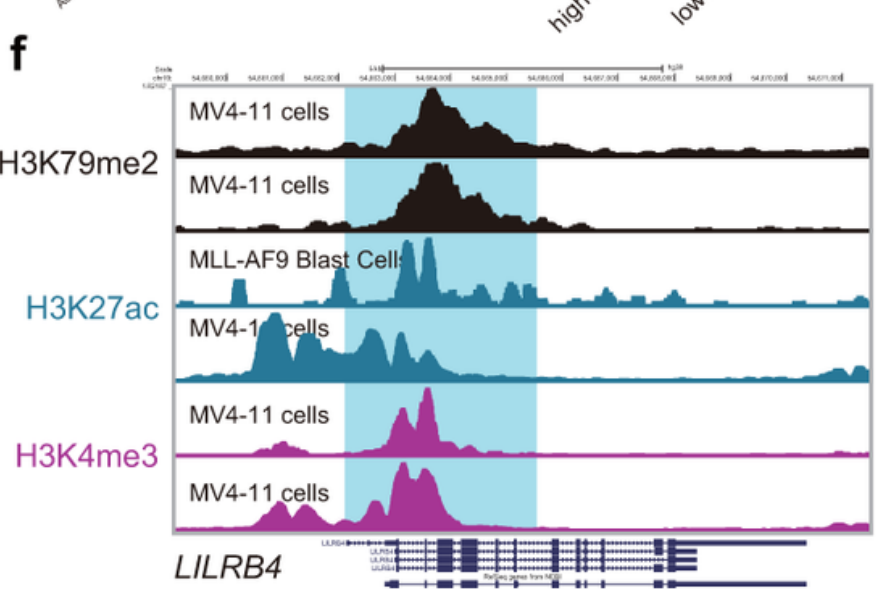

\section{Figure 6}

LILRB4 is aberrantly overexpressed in MLL-rearranged AML and is likely a direct target of MLL-fusion proteins. (a) Expression differences of LILRB genes in molecular subtypes of AML, and pre-B-ALL, DLBCL, and $\mathrm{MM}$. The expression fold change between each subtype and the remaining samples in the same 
disease were compared using the Wilcoxon rank sum test. The color of the dots indicates fold changes $(\log 2)$ and size indicates the FDR values. The FDR values were categorized into five groups based on significance cutoffs for visualization (0.05, 0.01, 0.001, 1e-5, 1e-16). (b) Bar plot showing LIRB4 expression (RNA-seq) in non-MLL-rearranged (HEL, MEG01, KASUMI1, KG1, NB4, K562, HL60, U937) and MLL-rearranged (SEM, MONOMAC6, NOMO1, RS411, MOLM13, THP1, MV411) cell lines from the CCLE database. The dotted line represents the mean expression of LILRB4 across all cell lines analyzed. (c) Comparison of LILRB4 expression among human primary AML cases with MLL rearrangements and those without MLL rearrangements in the BeatAML dataset. (d) Box plots showing ssGSEA scores of two MLL-related gene signatures (MULLIGHAN_MLL_SIGNATURE_1_DN and

MULLIGHAN_MLL_SIGNATURE_1_UP) between patients (TCGA dataset) with high and low LILRB4 expression. (e) ChIP-seq tracks for MLL-fusion proteins, H3K79me2, H3K27ac, and H3K4me3 at LILRB4 gene loci in MV4-11 and THP-1 cell lines. ChIP-seq data were obtained from GSE79899. (f) ChIP-seq tracks for H3K79me2, H3K27ac, and H3K4me3 at LILRB4 gene loci in MV4-11 and MLL-AF9 transformed blast cells. ChIP-seq data were obtained from GSE82116, GSE71779, GSE89336, GSE71776, and GSE61785.

\section{a}
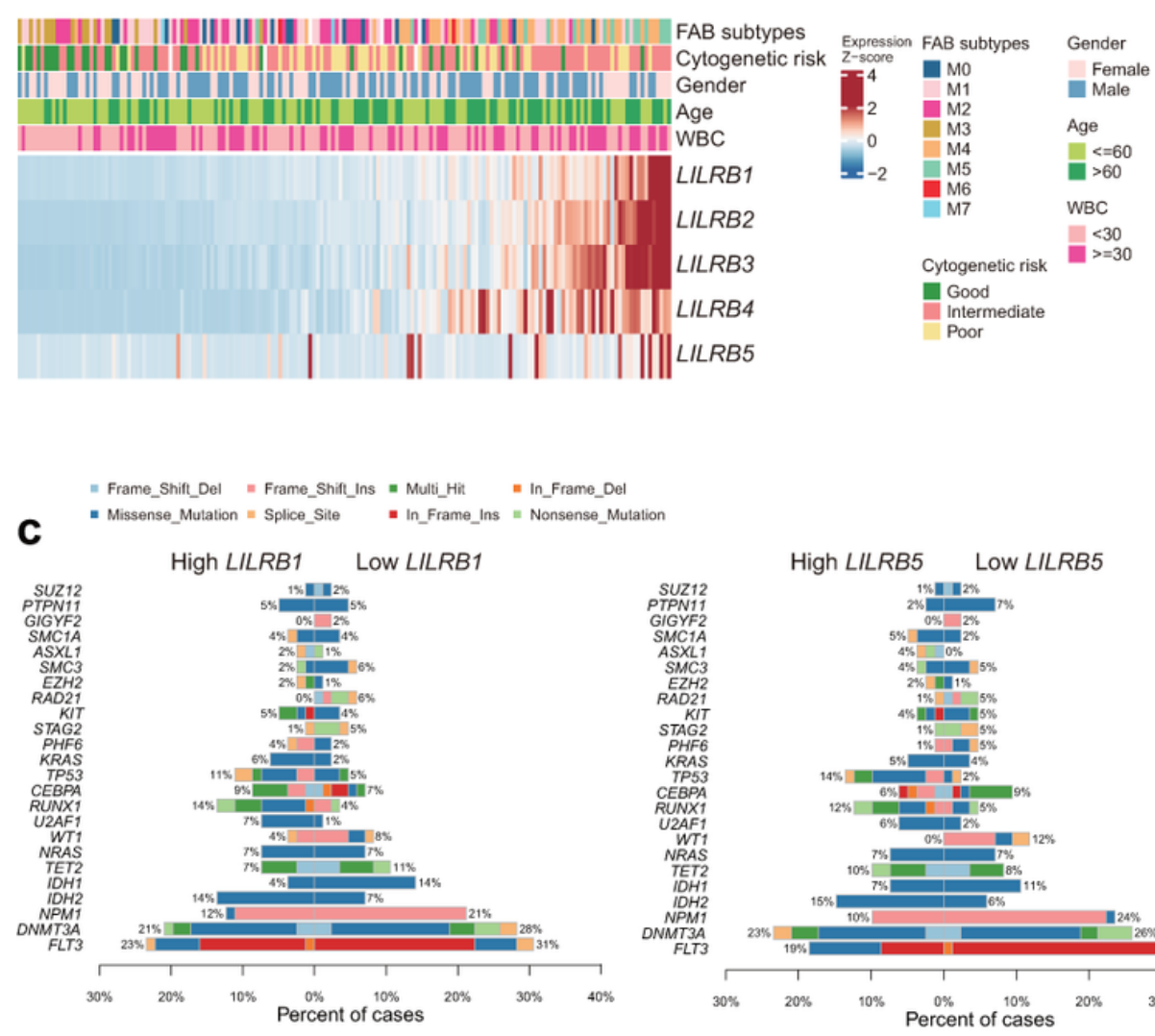

b

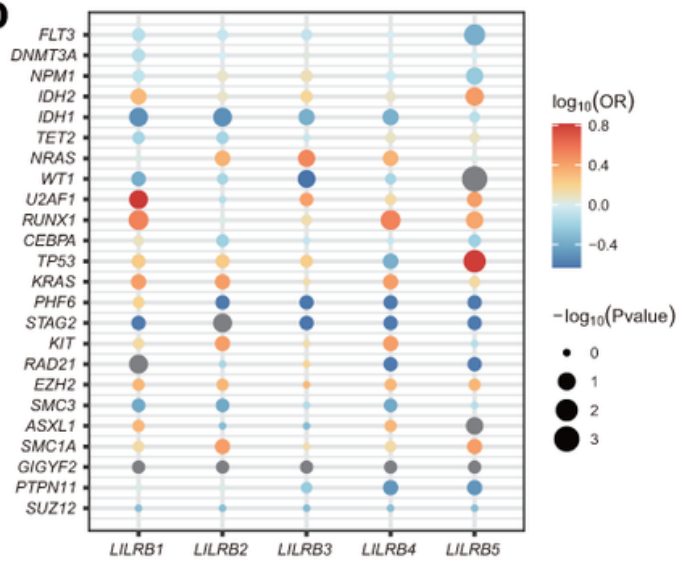

d

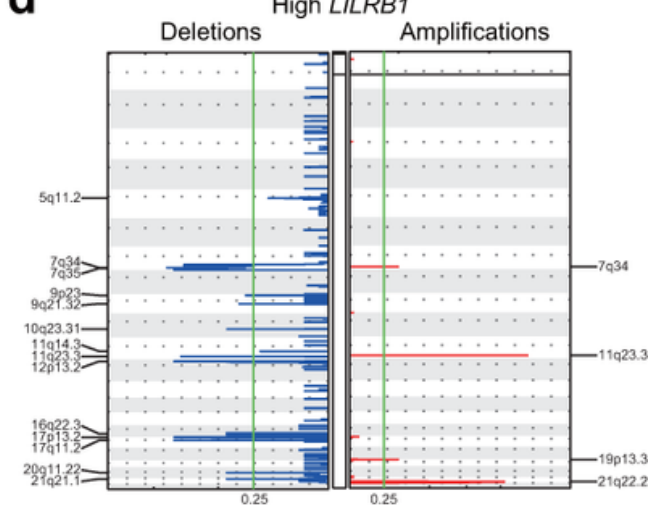

\section{Figure 7}

LILRBs expression correlate with distinct genomic alterations in AML. (a) Heatmap showing association between LILRBs expression and clinical characteristics in the TCGA AML cohort. (b) Bubble plot showing associations between the expression of LILRB1-LILRB5 and common mutational events in the TCGA dataset. Bubble size indicates - $\log 10$ (Fisher test p-value). Color signifies - $\log 10$ (odds ratio), positive 
association is indicated with red circles, negative with blue circles, and non-association with gray circles. (c) Co-bar plots showing the comparison of mutational profiles between patients with high and low LILRB1/LILRB5 expression in the TCGA dataset. (d) GISTIC analyses identified recurrent copy number alterations in AML patients with high LILRB1 expression.

a

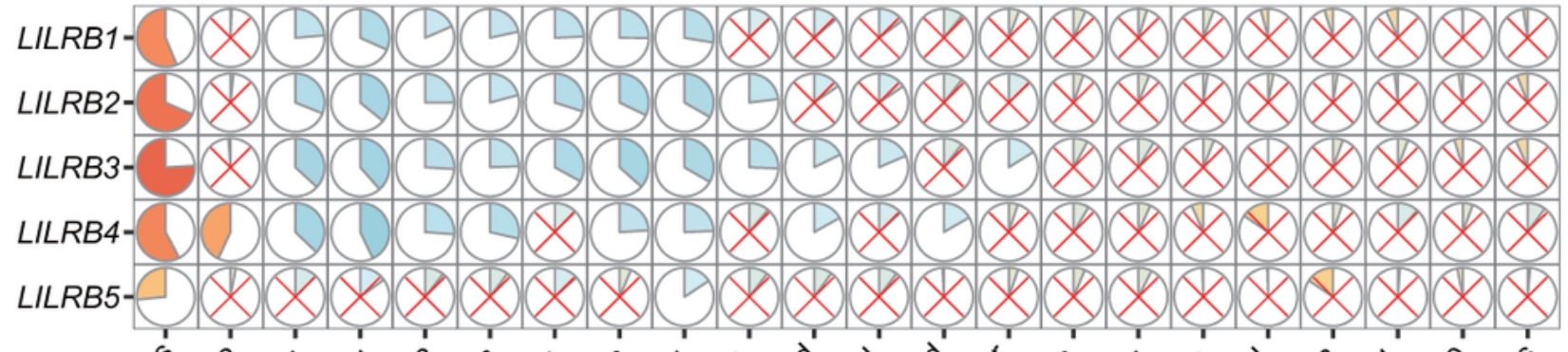

Correlation
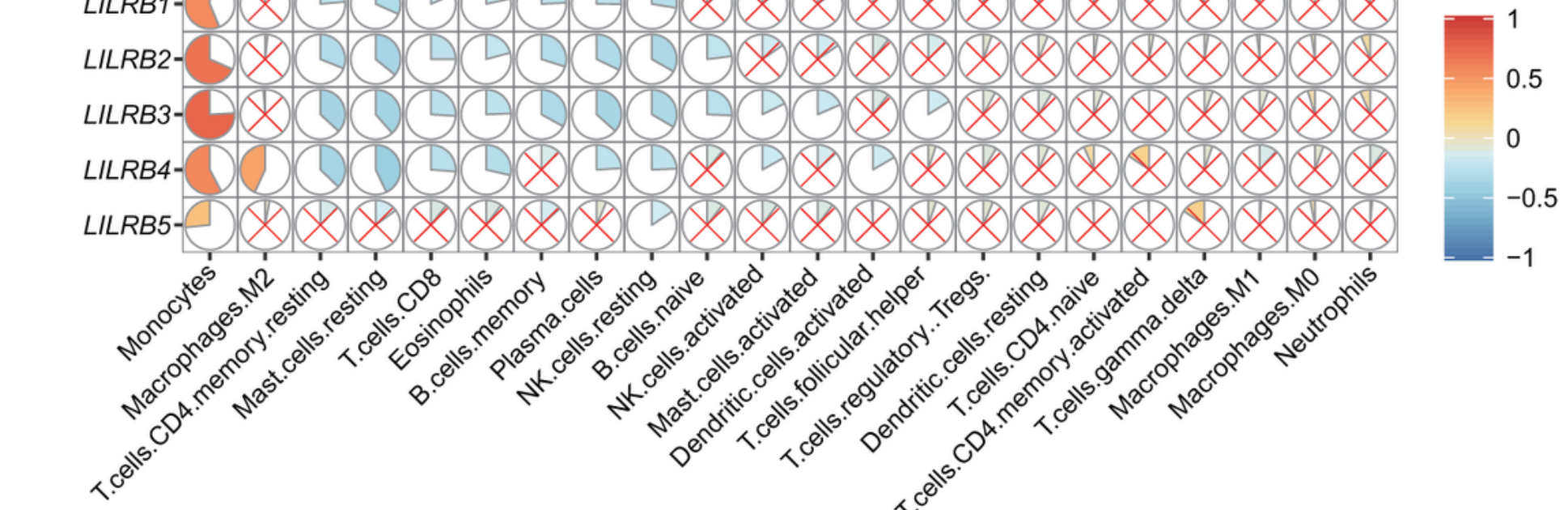

b
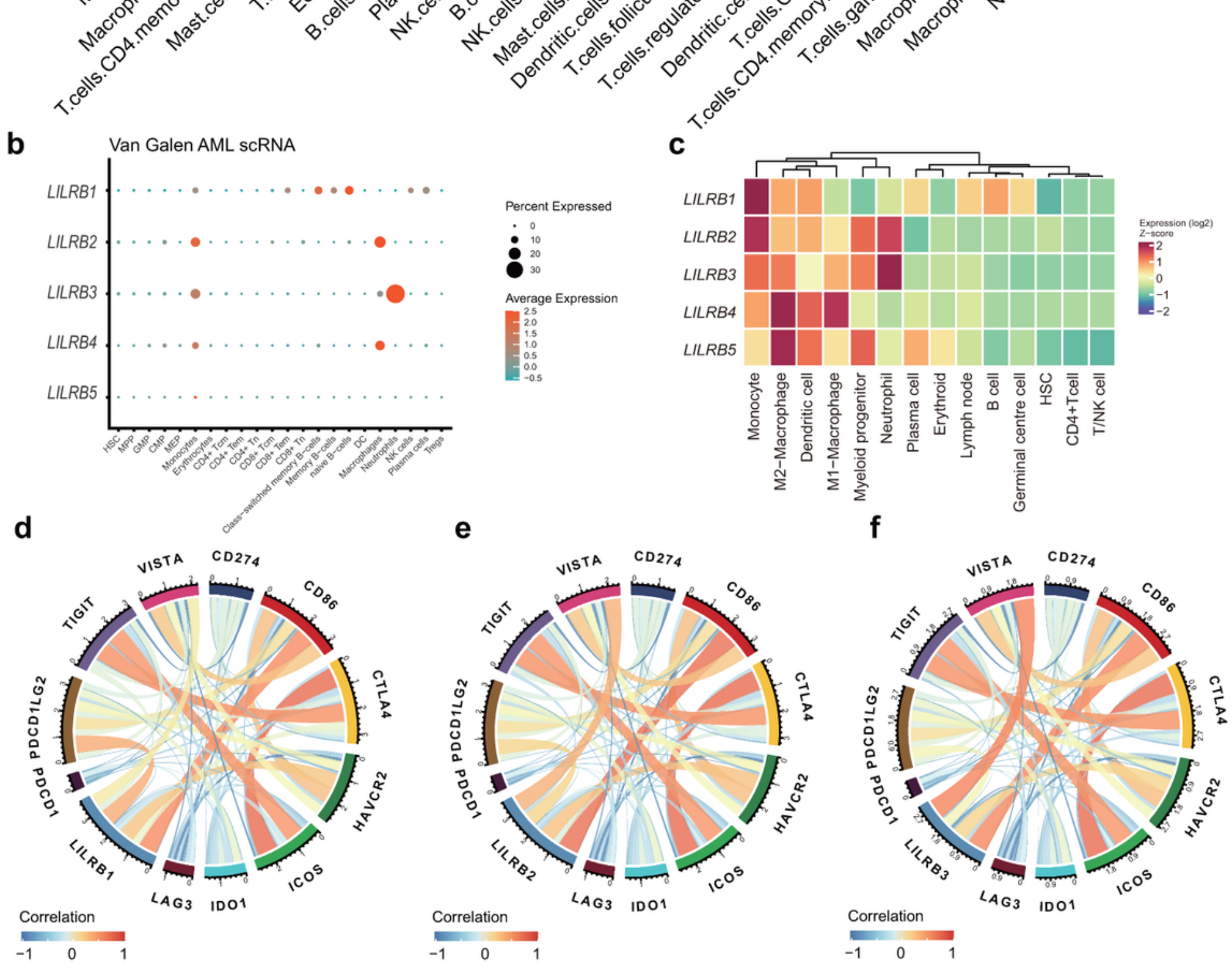

Figure 8 
The relation between LILRBs expression with immune cell infiltration and immune checkpoints. (a) Correlation matrix plot showing correlations between LILRBs and tumor immune infiltrating cells (TIICs). The overall immune cell compositions were estimated by CIBERSORT in the TCGA dataset. (b) Dot plot showing expression pattens of LILRBs in annotated cell types from 16 AML scRNA-seq samples (Van Galen AML scRNA). The color of the dots indicates average expression and size indicates percentage of cells with detectable expression. (c) Heatmap showing LILRBs expression in normal cell populations from the Hemap dataset. (d-f) Circos plots showing correlation between the expression of LILRB1 (d), LILRB2 (e), and LILRB3 (f) and immune checkpoint genes in the TCGA dataset.

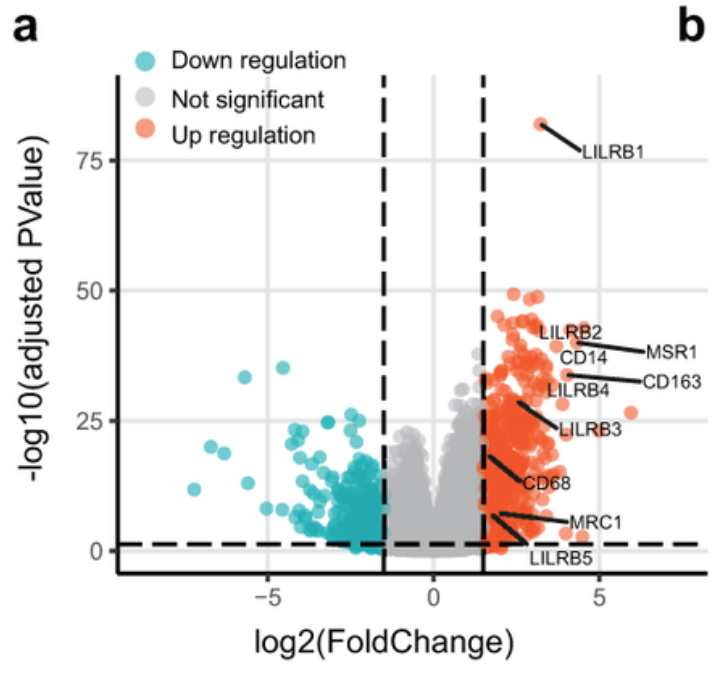

C

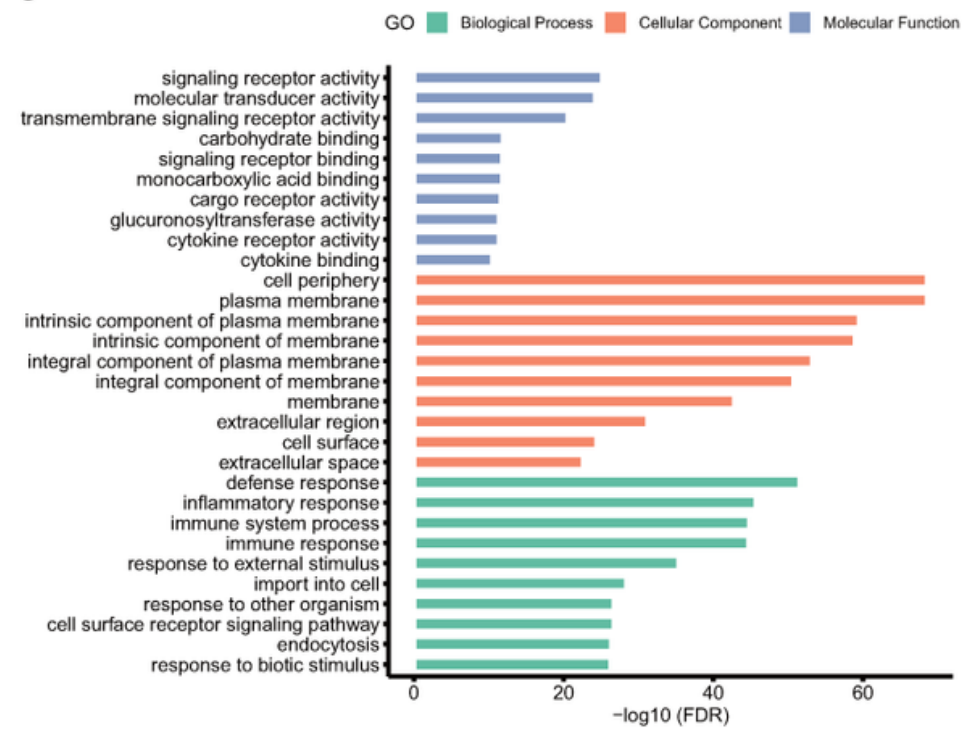

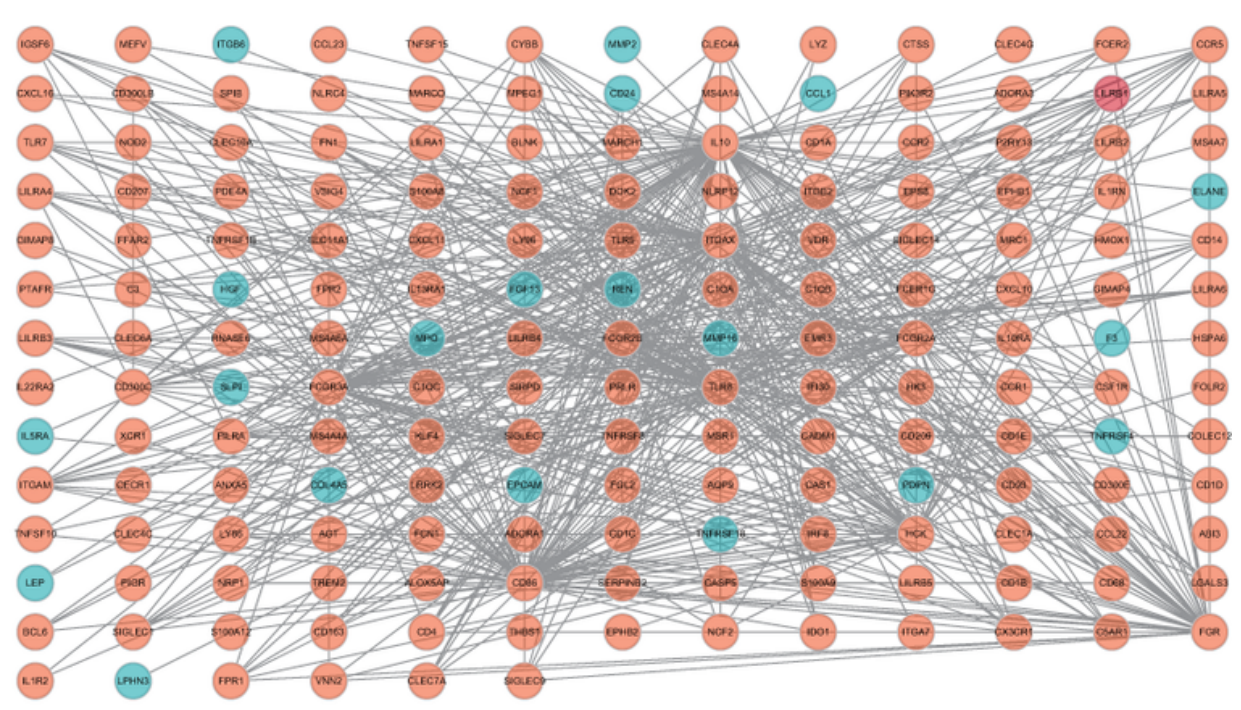

d

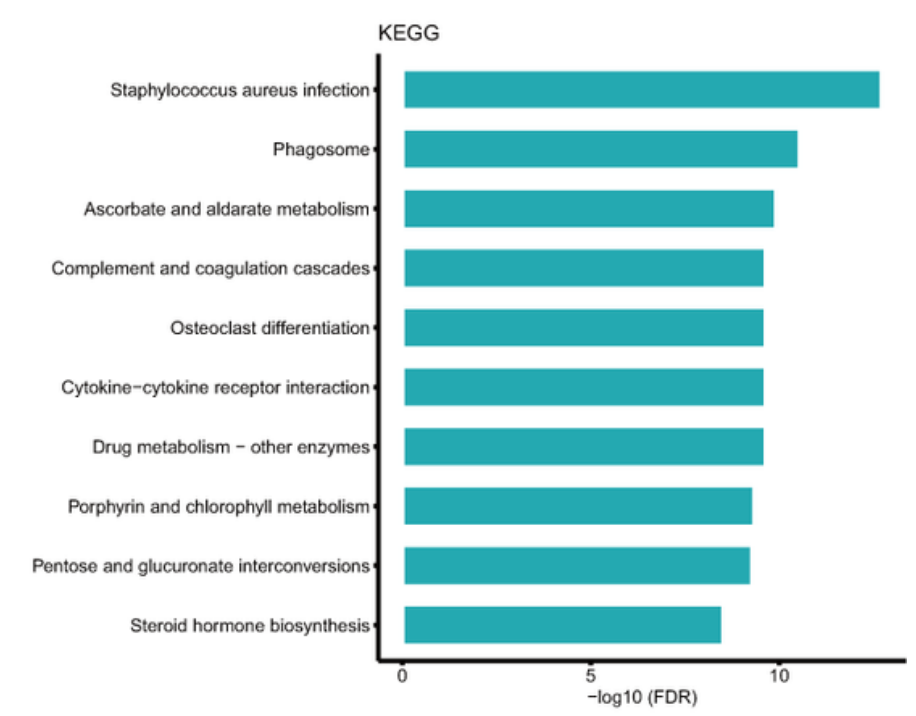

Figure 9

The biological significance of LILRBs expression in AML. (a) Volcano plot showing differentially expressed genes (DEGs) between high and low LILRB1 expressers. (b) Cytoscape analysis of LILRB1related network using PPI information obtained from STRING database (http://stringdb.org/). Red nodes represent up-regulated genes and blue represent down-regulated genes. (c and d) GO (c) and KEGG (d) analysis of DEGs. 
a

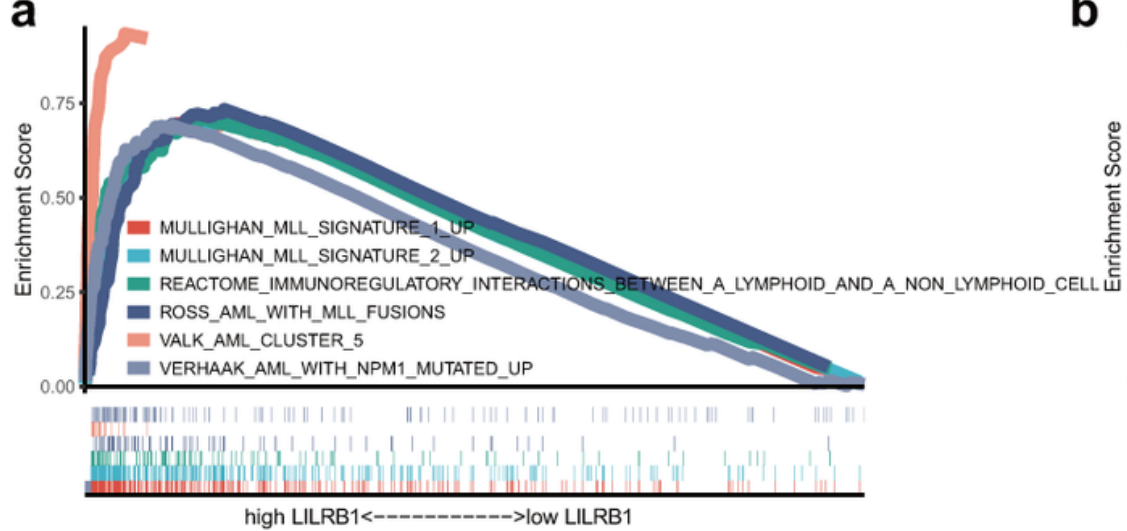

high LILRB1<------------>>low LILRB1

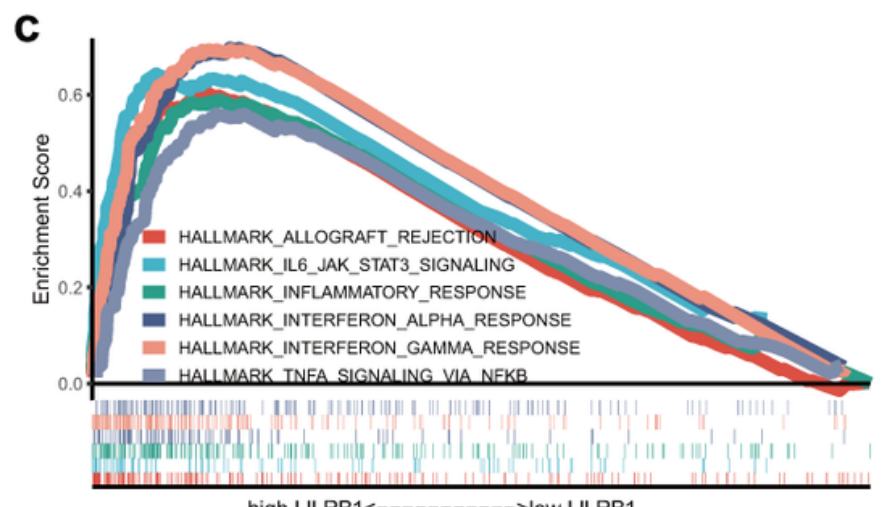

high LILRB1<------------->low LILRB1 b

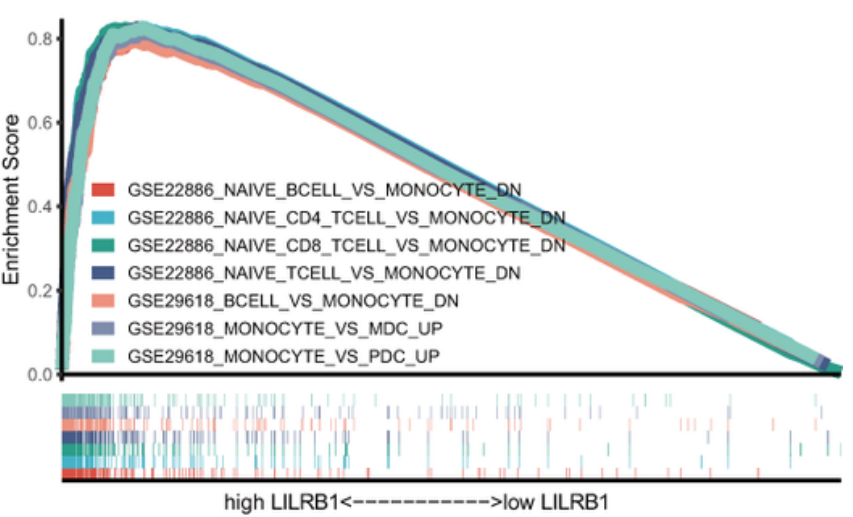

\section{Figure 10}

Gene set enrichment analysis (GSEA) of patients with high and low LILRB1 expression, with curated (a), immunologic (b), and hallmark (c) gene sets obtained from the Molecular Signatures Database (MSigDB).

\section{Supplementary Files}

This is a list of supplementary files associated with this preprint. Click to download.

- SupplementaryData1.xlsx

- SupplementaryData2.xlsx

- SupplementaryData3.xlsx

- Supplementarylnformation.docx 